\title{
Sliding mode control for a generalization of the Caginalp phase-field system
}

\author{
Pierluigi Colli and Davide Manini \\ Dipartimento di Matematica "F. Casorati", Università di Pavia \\ Via Ferrata, 1, 27100 Pavia, Italy \\ Email: pierluigi.colli@unipv.it davide.manini01@universitadipavia.it
}

\begin{abstract}
In the present paper, we present and solve the sliding mode control (SMC) problem for a secondorder generalization of the Caginalp phase-field system. This generalization, inspired by the theories developed by Green and Naghdi on one side, and Podio-Guidugli on the other, deals with the concept of thermal displacement, i.e., a primitive with respect to the time of the temperature. Two control laws are considered: the former forces the solution to reach a sliding manifold described by a linear constraint between the temperature and the phase variable; the latter forces the phase variable to reach a prescribed distribution $\varphi^{*}$. We prove existence, uniqueness as well as continuous dependence of the solutions for both problems; two regularity results are also given. We also prove that, under suitable conditions, the solutions reach the sliding manifold within finite time.
\end{abstract}

Key words. phase field system, nonlinear boundary value problems, phase transition, sliding mode control, state-feedback control law.

AMS subject classification. 35K55, 35B30, 80A22, 34H05, $93 \mathrm{~B} 52$.

\section{Introduction}

For many years, sliding mode control (SMC) has been recognized as one of the best approaches for the design of robust controllers for nonlinear dynamical systems. Nowadays, SMC is considered a standard tool for the regulation of time-evolving systems in finite dimension 3, 14, 16, 29, 31.

The design of feedback control systems with sliding modes involves the construction of suitable control functions enforcing motion along a given manifold of lower dimension, called sliding manifold. The main idea is (i) to identify this manifold where the control target is fulfilled and such that the original system restricted to this sliding manifold has a desired behavior; (ii) to act on the system through a suitable control term in order to constrain the evolution on it. This new term forces the trajectories of the system to reach the sliding manifold and maintains them along it.

Sliding mode controls feature robustness with respect to unmodelled dynamics and insensitivity to external disturbances. At the same time they are relatively easy to design. For these reasons, in the last years there has been a growing interest in bringing these methods for finite-dimensional systems described by ODEs 20,23,24 to the realm of PDEs. While certain early works going in this direction 22, 24, 25, deal with particular classes of PDEs, the theoretical development in a general Hilbert space setting has gained attention only in the last years [10,21,30. We also point out the article 2, mainly related to the contents of this paper, and the subsequent contributions 9, 11,12, also dealing with systems of PDEs.

In this paper, the considered system describes the spatial and time fluctuations close to a phase transition. In order to take into account the effects of phase dissipation, Caginalp introduced [6] a phase-field system consisting of the following equations

$$
\begin{aligned}
& (\vartheta+l \varphi)_{t}-\kappa \Delta \vartheta=f, \quad \text { in } \Omega \times(0, T), \\
& \varphi_{t}-\Delta \varphi+F^{\prime}(\varphi)=\gamma \vartheta, \quad \text { in } \Omega \times(0, T) .
\end{aligned}
$$

Here, $\Omega \subset \mathbb{R}^{N}$ represents the spatial domain where the evolution takes place and $T>0$ is the final time of the evolution. While the case $N=3$ is the one of physical interest, we will carry out our analysis for 
all $N$. A usual choice, at least for the phase variable $\varphi$, is to complement the equations with standard homogeneous Neumann boundary conditions $\partial_{n} \varphi=\partial_{n} \vartheta=0$ on $\partial \Omega \times(0, T)$, plus the initial conditions $\vartheta(\cdot, 0)=\vartheta_{0}$ and $\varphi(\cdot, 0)=\varphi_{0}$.

The variable $\vartheta$ represents the relative temperature, i.e. the difference between the actual temperature and the fixed critical temperature for the phase transition. The variable $\varphi$ has the meaning of a phase parameter: $\varphi<0$ indicates one of the two phases; $\varphi>0$ indicates the other phase; $\varphi(x, t)=0$ usually indicates that position $x$ is at the interface between the two phases at time $t . F^{\prime}$ is the derivative of a double-well potential $F$. A few examples for the double-well potential $F$ are

$$
\begin{aligned}
& F(r)=\frac{1}{4}\left(r^{2}-1\right)^{2}, \\
& F(r)= \begin{cases}(1+r) \log (1+r)+(1-r) \log (1-r)-\left(c_{0}+1\right) r^{2}, & \text { if }|r|<1 \\
2 \log 2-c_{0}-1, & \text { if }|r|=1 \\
+\infty & \text { otherwise, }\end{cases} \\
& F(r)= \begin{cases}-c_{0} r^{2}, & \text { if }|r| \leq 1, \\
+\infty, & \text { otherwise }\end{cases}
\end{aligned}
$$

where $c_{0} \in \mathbb{R}, c_{0}>0$. Please note that the potential in (1.4) has a derivative in $(-1,1)$ becoming singular as $r$ approaches -1 or 1 , while the potential (1.5) is non-smooth so that part of its derivative should be replaced by the subdifferential of the indicator function of $[-1,1]$.

The physical equations originating the system (1.1)-1.2) art

$$
\begin{aligned}
& \partial_{t} e+\operatorname{div} \mathbf{q}=\tilde{f}, \\
& \partial_{t} \varphi+\frac{\delta \mathcal{F}}{\delta \varphi}=0,
\end{aligned}
$$

where $e$ denotes the internal energy, q the thermal flux, and $\tilde{f}$ the heat source. The term $\frac{\delta \mathcal{F}}{\delta \varphi}$ represents the variational derivative with respect to $\varphi$ of the following functional

$$
\mathcal{F}(\vartheta, \varphi)=\int_{\Omega}\left(-\frac{c_{0}}{2} \vartheta^{2}-\gamma \vartheta \varphi+F(\varphi)+\frac{1}{2}|\nabla \varphi|^{2}\right),
$$

where the constants $c_{0}$ and $\gamma$ represent the specific heat and the latent heat coefficient, respectively. Note that the term $-\gamma \vartheta \varphi$ favors states having concordant relative temperature and phase variable. The internal energy $e$ may be derived from the functional $\mathcal{F}$, taking minus its variational derivative with respect to $\vartheta$, i.e.,

$$
e=-\frac{\delta \mathcal{F}}{\delta \vartheta}=c_{0} \vartheta+\gamma \varphi
$$

Equation (1.7) yields equation (1.2) by standard variational derivative taking into account the homogeneous Neumann condition for $\varphi$. We set $l:=\gamma / c_{0}$ and $f:=\tilde{f} / c_{0}$. If we assume the classic Fourier law

$$
\mathbf{q}=-c_{0} \kappa \nabla \vartheta
$$

equation (1.6) yields (1.1). The homogeneous Neumann condition for $\vartheta$ follows from the no-flux condition $\mathbf{q} \cdot \mathbf{n}=0$ on the boundary of $\Omega$.

A sliding-mode analysis has been carried out recently for the system described by the equations (1.1)(1.2) 2. In the quoted paper three cases are taken into consideration (labeled as Problem A-C). In Problem A, the sliding manifold is given by a linear constraint between $\vartheta$ and $\varphi$; in Problems B and $\mathrm{C}$ the phase $\varphi$ is forced to reach a prescribed phase distribution $\varphi^{*}$. While in Problems A and B the control law is non-local in the spatial variable, in Problem $\mathrm{C}$ the control term is fully local.

In the present paper we carry out a similar sliding-mode analysis, for modified equations where the Fourier law (1.10) is generalized in the light of the works by Green and Naghdi [17. 19] and (more recently) by Podio-Guidugli [26, referring to Thermodynamics. These papers introduced the notion of thermal displacement, which is a primitive of the temperature, i.e.

$$
w(x, t)=w_{0}(x)+\int_{0}^{t} \vartheta(x, s) \mathrm{d} s
$$

\footnotetext{
${ }^{1}$ Please note that in this paper we will use both the notations $p_{t}$ and $\partial_{t} p$ to denote the derivative of a function $p$.
} 
where $w_{0}$ represents a given datum accounting for a possible previous thermal history of the phenomenon. Making use of this new variable, these authors proposed three theories for heat transmission labeled as type I-III. Type I theory, after suitable linearization, yields the standard Fourier law

$$
\mathbf{q}=-c_{0} \kappa \nabla w_{t} \quad(\text { type I), }
$$

which has been studied extensively. Linearized versions of type II and III give the following heatconduction laws

$$
\begin{array}{ll}
\mathbf{q}=-c_{0} \tau \nabla w & \text { (type II), } \\
\mathbf{q}=-c_{0} \kappa \nabla w_{t}-c_{0} \tau \nabla w & \text { (type III). }
\end{array}
$$

It is important to note that the thermal displacement $w$ becomes necessary to describe type II and III laws, whereas type I law can be described just in terms of the temperature $\vartheta=\partial_{t} w$. The role of the primitive $w$ in type II and III theories is to account for the past thermal history of the heat-conducting body.

This paper focuses on the most general type III theory. In type III theory, the special $\tau=0$ case reduces to standard type I theory; $\kappa=0$ yields type II theory. Equation (1.6), along with type III law (1.14), leads to this formulation

$$
\left(w_{t}+l \varphi\right)_{t}-\kappa \Delta w_{t}-\tau \Delta w=f, \quad \text { in } \Omega \times(0, T) .
$$

Equation (1.2) with the substitution $\vartheta=\partial_{t} w$ becomes

$$
\varphi_{t}-\triangle \varphi+F^{\prime}(\varphi)=\gamma w_{t}, \quad \text { in } \Omega \times(0, T) .
$$

The no-flux condition $\mathbf{q} \cdot \mathbf{n}=0$ generates the homogeneous Neumann boundary condition $\partial_{n} w=0$. For the system (1.15)-(1.16), well-posedness, asymptotic analysis, and convergence of the solutions as $\tau \rightarrow 0$ to the solution of the original Caginalp system (1.1)-(1.2) has been carried out in [7, 8 .

In order to enable the SMC in the system above, we add a feedback term in either equation (1.15) or (1.16) in order to force the solutions $(w(t), \varphi(t))$ to reach the sliding manifold. In the present paper, we consider two cases. As for the first one, we adopt the following linear condition connecting $w$ and $\varphi$

$$
\partial_{t} w(t)+\alpha \varphi(t)=\eta^{*}
$$

to describe the sliding manifold, and the feedback control is added to the left-hand side of equation (1.15). In (1.17), $\alpha$ is a real positive constant and $\eta^{*}$ a prescribed function independent of time. For the second case, our aim is that the phase reaches a prescribed phase distribution $\varphi^{*}$; in order to achieve that, we insert the feedback term in (1.16). The linear condition (1.17), as well as the choice of the sign operator in $L^{2}(\Omega)$, corresponds to the Problem (A) in [2] for the first case studied there, whereas the second case we investigate here corresponds to the Problem (B) in [2]. However, with respect to the arguments used in [2], here we adopt a slightly different approach, based on the simplification of the auxiliary lemma and on the observation that the Moreau-Yosida regularization of the norm of a Banach space converges uniformly. Thus, we improve some technical aspects of [2]. Moreover, let us point out that our system (1.15) - (1.16) is more difficult to handle, due to the hyperbolicity of equation (1.15).

This paper is organized as follows. The next section deals with the common notation and the considered system of equations; it also contains the precise statements of results. Our theorems establish well-posedness, regularity properties for the solutions, and in particular the existence of sliding modes for both problems. We note that in our argumentation the results are grouped by problem. The remaining sections, from $\S 3$ to $\S 6$, are devoted to the proofs.

\section{Common notation and main results}

In this section, we set the notation and present the problems that we will solve, as well as the results concerning well-posedness of the problems and regarding SMC. Moreover, a few technical tools are recalled.

First of all, we require for $\Omega \subset \mathbb{R}^{N}$ to be an open, bounded, smooth set. $\Gamma$ and $\partial_{n}$ represent the boundary of $\Omega$ and the outward normal derivative on $\Gamma$, respectively. We set $Q_{t}=\Omega \times(0, t)$ for $t \in(0, T]$ and $Q=Q_{T}$.

In the sequel, we will make use of techniques of convex analysis, so we split the potential $F$ as the sum $\widehat{\beta}+\widehat{\pi}$, by requiring that

$$
\begin{aligned}
& \widehat{\beta}: \mathbb{R} \rightarrow[0,+\infty] \text { is convex, proper, l.s.c. with } \widehat{\beta}(0)=0, \\
& \widehat{\pi}: \mathbb{R} \rightarrow \mathbb{R} \text { is } C^{1} \text { and } \widehat{\pi}^{\prime} \text { is Lipschitz-continuous. }
\end{aligned}
$$


We define $\beta$ and $\pi$ as the subdifferential [27, $\S 23$ ] of $\widehat{\beta}$ and the derivative of $\widehat{\pi}$, respectively. It turns out that $\beta$ is a maximal monotone graph of $\mathbb{R}^{2}$, such that $0 \in \beta(0)$. We indicate with $\beta^{\circ}(r)$ the element of $\beta(r)$ having minimum modulus.

We make the following assumptions on the data of the problem

$$
\begin{aligned}
& \kappa, \tau, \gamma, l \in(0,+\infty), \\
& f \in L^{2}(Q) .
\end{aligned}
$$

We introduce the following Hilbert spaces

$$
H:=L^{2}(\Omega), \quad V:=H^{1}(\Omega), \quad W:=\left\{v \in H^{2}(\Omega): \partial_{n} v=0\right\} .
$$

On $H$ and $V$ we put the standard Hilbert norm, while we endow $W$ with the norm $\|u\|_{W}^{2}=\|u\|_{H}^{2}+\|\triangle u\|_{H}^{2}$, which is equivalent to the norm $\|\cdot\|_{H^{2}(\Omega)}$, by the smooth boundary condition and elliptic regularity (see e.g. [5. $\S 9.6]$ or [15, $\S 6.3])$. The norm $\|\cdot\|_{H}$ will also denote the norm of the space $H^{N}=L^{2}\left(\Omega ; \mathbb{R}^{N}\right)$. The scalar product of $H$ and $H^{N}$ will be denoted with $(\cdot, \cdot)$. We define the Sign operator for the Hilbert space $H$ as the subdifferential of the norm $\|\cdot\|_{H}$, namely

$$
\operatorname{Sign}(v)= \begin{cases}\frac{v}{\|v\|_{H}} & \text { if } v \neq 0 \\ B_{H} & \text { if } v=0\end{cases}
$$

where $B_{H}$ is the closed unit ball of $H$. The regularity hypotheses for the initial data are

$$
\vartheta_{0} \in V, \quad w_{0} \in W, \quad \varphi_{0} \in V, \quad \widehat{\beta}\left(\varphi_{0}\right) \in L^{1}(\Omega) .
$$

A solution is a quadruplet $(w, \varphi, \xi, \sigma)$, for which we require, at least, the following regularity

$$
\begin{aligned}
& w \in H^{2}(0, T ; H) \cap W^{1, \infty}(0, T ; V) \cap H^{1}(0, T ; W), \\
& \varphi \in H^{1}(0, T ; H) \cap L^{\infty}(0, T ; V) \cap L^{2}(0, T ; W), \\
& \xi \in L^{2}(0, T ; H), \\
& \sigma \in L^{\infty}(0, T ; H) .
\end{aligned}
$$

We note that the Neumann boundary condition $\partial_{n} w=\partial_{n} \varphi=0$ is incorporated in the definition of the space $W$.

Given a sliding mode parameter $\rho>0, \alpha \in \mathbb{R}$ and a target function $\eta^{*}$ the first problem is to find a quadruplet $(w, \varphi, \xi, \sigma)$ satifying the regularity conditions (2.6)-(2.9) and

$$
\begin{aligned}
& \left(w_{t}+l \varphi\right)_{t}-\kappa \Delta w_{t}-\tau \Delta w+\rho \sigma=f \quad \text { a.e. in } Q, \\
& \sigma \in \operatorname{Sign}\left(w_{t}+\alpha \varphi-\eta^{*}\right) \quad \text { in } H, \text { a.e. in }(0, T), \\
& \varphi_{t}-\Delta \varphi+\xi+\pi(\varphi)=\gamma w_{t} \quad \text { a.e. in } Q, \\
& \xi \in \beta(\varphi) \quad \text { a.e. in } Q, \\
& w_{t}(0)=\vartheta_{0}, \quad w(0)=w_{0}, \quad \varphi(0)=\varphi_{0} \quad \text { a.e. in } \Omega .
\end{aligned}
$$

We label the problem above as Problem (A). The regularity hypothesis for the target function $\eta^{*}$ is

$$
\eta^{*} \in W \text {. }
$$

Let us emphasize that the feedback law is highly non-local, as the value of the feedback term at $(x, t)$ depends on $(w(\cdot, t), \varphi(\cdot, t))$ and not only on $(w(x, t), \varphi(x, t))$. The sliding-mode parameter $\rho>0$ represents the strength of the control law and it plays a central role in this kind of analysis. Accordingly, we will highlight the dependence on $\rho$ in all our estimates.

On the other hand, given $\rho>0$ and a target function $\varphi^{*}$, Problem (B) consists in finding a quadruplet $(w, \varphi, \xi, \sigma)$ satifying the regularity conditions (2.6)-(2.9) and

$$
\begin{aligned}
& \left(w_{t}+l \varphi\right)_{t}-\kappa \Delta w_{t}-\tau \Delta w=f \quad \text { a.e. in } Q, \\
& \varphi_{t}-\Delta \varphi+\xi+\pi(\varphi)+\rho \sigma=\gamma w_{t} \quad \text { a.e. in } Q, \\
& \sigma \in \operatorname{Sign}\left(\varphi-\varphi^{*}\right) \quad \text { in } H, \text { a.e. in }(0, T), \\
& \xi \in \beta(\varphi) \quad \text { a.e. in } Q, \\
& w_{t}(0)=\vartheta_{0}, \quad w(0)=w_{0}, \quad \varphi(0)=\varphi_{0} \quad \text { a.e. in } \Omega .
\end{aligned}
$$

For this problem, the regularity required for $\varphi^{*}$ is

$$
\varphi^{*} \in W \quad \text { and } \quad \beta^{\circ}\left(\varphi^{*}\right) \in H .
$$


Results for Problem (A). First of all we present the well-posedness results, starting from the existence theorem.

Theorem 2.1 (Existence). Assume (2.1)-(2.5), and (2.15). Then there exist two constants $C_{1}, C_{2}>0$ such that for every $\rho>0$ the problem (2.10)-(2.14) has at least a solution $(w, \varphi, \xi, \sigma)$ satisfying (2.6) -(2.9) and the estimates

$$
\begin{aligned}
& \|w\|_{W^{1, \infty}(0, T ; V) \cap H^{1}(0, T ; W)}+\|\varphi\|_{H^{1}(0, T ; H) \cap L^{\infty}(0, T ; V) \cap L^{2}(0, T ; W)} \\
& +\rho\left\|w_{t}+\alpha \varphi-\eta^{*}\right\|_{L^{1}(0, T ; H)}+\|\widehat{\beta}(\varphi)\|_{L^{\infty}\left(0, T ; L^{1}(\Omega)\right)} \\
& +\|\xi\|_{L^{2}(0, T ; H)}+\|\sigma\|_{L^{\infty}(0, T ; H)} \leq C_{1}, \\
& \|w\|_{H^{2}(0, T ; H)} \leq C_{2}\left(1+\rho^{1 / 2}\right) .
\end{aligned}
$$

The following result gives further regularity of the solutions under the hypothesis

$$
\varphi_{0} \in W \quad \text { and } \quad \beta^{\circ}\left(\varphi_{0}\right) \in H .
$$

Theorem 2.2 (Further regularity). Assume the hypotheses of Theorem 2.1 and the condition (2.24). Then every solution $(w, \varphi, \xi, \sigma)$ given by Theorem 2.1 satisfies

$$
\begin{gathered}
\varphi \in W^{1, \infty}(0, T ; H) \cap H^{1}(0, T ; V) \cap L^{\infty}(0, T ; W), \\
\xi \in L^{\infty}(0, T ; H),
\end{gathered}
$$

and

$$
\|\varphi\|_{W^{1, \infty}(0, T ; H) \cap H^{1}(0, T ; V) \cap L^{\infty}(0, T ; W)}+\|\xi\|_{L^{\infty}(0, T ; H)} \leq C_{3}\left(1+\rho^{1 / 2}\right),
$$

where $C_{3}>0$ is a constant independent of $\rho$.

The regularity given by the above theorem is necessary for proving the existence of sliding modes.

The next result closes the topic of the well-posedness of the problem. As consequence, we have that under the assumption $l=\alpha$ the solution is unique.

Theorem 2.3 (Continuous dependence). Suppose (2.1)-(2.3), (2.15), $\rho>0$, and $l=\alpha$. Let $i=1,2$. We consider $\left(\vartheta_{0, i}, w_{0, i}, \varphi_{0, i}, f_{i}, w_{i}, \varphi_{i}, \xi_{i}, \sigma_{i}\right)$, where the functions $\left(\vartheta_{0, i}, w_{0, i}, \varphi_{0, i}\right)$ are initial data satifying equation (2.5), $f_{i}$ is a function satisfying (2.4), and $\left(w_{i}, \varphi_{i}, \xi_{i}, \sigma_{i}\right)$ is a solution for Problem (B) given by Theorem 2.1 with $\left(\vartheta_{0}, w_{0}, \varphi_{0}\right)=\left(\vartheta_{0, i}, w_{0, i}, \varphi_{0, i}\right)$ and $f=f_{i}$. Then, there exists a constant $C_{4}>0$, independent of $\vartheta_{0, i}, w_{0, i}, \varphi_{0, i}, f_{i}$, and $\rho$, such that

$$
\begin{aligned}
& \left\|w_{1}-w_{2}\right\|_{W^{1, \infty}(0, T ; H) \cap H^{1}(0, T ; V)}+\left\|\varphi_{1}-\varphi_{2}\right\|_{L^{\infty}(0, T ; H) \cap L^{2}(0, T ; V)} \\
& \leq C_{4}\left(\left\|\vartheta_{0,1}-\vartheta_{0,1}\right\|_{H}+\left\|w_{0,1}-w_{0,2}\right\|_{V}\right. \\
& \left.+\left\|\varphi_{0,1}-\varphi_{0,2}\right\|_{H}+\left\|f_{1}-f_{2}\right\|_{L^{2}(Q)}\right) .
\end{aligned}
$$

Corollary 2.4 (Uniqueness). Suppose that the hypotheses of Theorem 2.1 hold true and that $l=\alpha$. Then the solution is unique.

Finally we come to the most important result for Problem (A): the theorem that guarantees that the solutions reach the sliding manifold in a finite time.

Theorem 2.5 (Sliding mode). Assume (2.1)-(2.4), (2.5), (2.15) (2.24), and $f \in L^{\infty}(0, T ; H)$. Then there exist $\rho^{*}>0$, such that the following condition is fulfilled: for every $\rho>\rho^{*}$ and for every solution $(w, \varphi, \xi, \sigma)$ to the problem (2.10)-(2.14) there exist a time $T^{*} \in[0, T)$, such that

$$
w_{t}(t)+\alpha \varphi(t)=\eta^{*}, \quad \text { a.e. in } \Omega \text {, for a.a. } t \in\left(T^{*}, T\right) .
$$

Remark 2.6. The statement of the above theorem gives no estimates for $\rho^{*}$ and $T^{*}$, but in the proof we will find certain bounds which we summarize here. Define $C_{5}$ and $\psi_{0}$ as

$$
\begin{aligned}
& C_{5}=\tau C_{1}+(\kappa \alpha+|\alpha-l|) C_{3}+\kappa\left\|\Delta \eta^{*}\right\|_{H}+\|f\|_{L^{\infty}(0, T ; H)}, \\
& \psi_{0}=\left\|\vartheta_{0}+\alpha \varphi_{0}-\eta^{*}\right\|_{H},
\end{aligned}
$$

where the constants $C_{1}$ and $C_{3}$ are given by Theorems 2.1 and 2.2 respectively. The quantity $\psi_{0}$ measures how far the initial state is from the sliding manifold. We will see that it is sufficient to choose

$$
\rho^{*}=2\left(\frac{\psi_{0}}{T}+C_{5}+\frac{C_{5}^{2}}{2}\right)
$$

to fulfill the condition described by the theorem. Moreover, for a given $\rho>\rho^{*}$, we will prove the following bound on $T^{*}$ :

$$
T^{*} \leq \frac{2 \psi_{0}}{\rho-2 C_{5}-C_{5}^{2}}<T .
$$


Results for Problem (B). For Problem (B) we have two theorems regarding well-posedness.

Theorem 2.7 (Existence). Assume (2.1)-(2.5) and (2.21). Then there exist two constants $C_{6}, C_{7}>0$, such that for every $\rho>0$ the problem (2.16)-(2.20) has at least a solution $(w, \varphi, \xi, \sigma)$ satisfying (2.6)-(2.9) and the estimates

$$
\begin{aligned}
& \|w\|_{W^{1, \infty}(0, T ; H) \cap H^{1}(0, T ; V)}+\|\varphi\|_{L^{\infty}(0, T ; H) \cap L^{2}(0, T ; V)}+\|\sigma\|_{L^{\infty}(0, T ; H)} \\
& +\rho\left\|\varphi-\varphi^{*}\right\|_{L^{1}(0, T ; H)} \leq C_{6}, \\
& \|w\|_{H^{2}(0, T ; H) \cap W^{1, \infty}(0, T ; V) \cap H^{1}(0, T ; W)}+\|\varphi\|_{H^{1}(0, T ; H) \cap L^{\infty}(0, T ; V) \cap L^{2}(0, T ; W)} \\
& +\|\xi+\rho \sigma\|_{L^{2}(0, T ; H)}+\|\widehat{\beta}(\varphi)\|_{L^{\infty}\left(0, T ; L^{1}\right)} \\
& +\rho\left\|\varphi(t)-\varphi^{*}\right\|_{L^{\infty}(0, T ; H)} \leq C_{7}\left(1+\rho^{1 / 2}\right) .
\end{aligned}
$$

Moreover, the components $w$ and $\varphi$ of the solution are uniquely identified. Furthermore, the components $\xi$ and $\sigma$ are uniquely identified as well, provided that $\beta$ is single-valued.

Theorem 2.8 (Continuous dependence). Suppose (2.1)-(2.3), (2.21), $\rho>0$, and $l=\alpha$. Let $i=1,2$. We consider $\left(\vartheta_{0, i}, w_{0, i}, \varphi_{0, i}, f_{i}, w_{i}, \varphi_{i}, \xi_{i}, \sigma_{i}\right)$, where the functions $\left(\vartheta_{0, i}, w_{0, i}, \varphi_{0, i}\right)$ are initial data satifying equation (2.5), $f_{i}$ is a function satisfying (2.4), and $\left(w_{i}, \varphi_{i}, \xi_{i}, \sigma_{i}\right)$ is a solution for Problem (B) given by Theorem 2.7 with $\left(\vartheta_{0}, w_{0}, \varphi_{0}\right)=\left(\vartheta_{0, i}, w_{0, i}, \varphi_{0, i}\right)$ and $f=f_{i}$. Then, there exists a constant $C_{8}>0$, independent of $\vartheta_{0, i}, w_{0, i}, \varphi_{0, i}, f_{i}$, and $\rho$, such that

$$
\begin{aligned}
& \left\|w_{1}-w_{2}\right\|_{W^{1, \infty}(0, T ; H) \cap H^{1}(0, T ; V)}+\left\|\varphi_{1}-\varphi_{2}\right\|_{L^{\infty}(0, T ; H) \cap L^{2}(0, T ; V)} \\
& \leq C_{8}\left(\left\|\vartheta_{0,1}-\vartheta_{0,1}\right\|_{H}+\left\|w_{0,1}-w_{0,2}\right\|_{V}+\left\|\varphi_{0,1}-\varphi_{0,2}\right\|_{H}+\left\|f_{1}-f_{2}\right\|_{L^{2}(Q)}\right) .
\end{aligned}
$$

For the sake of completeness, we give a regularity result similar to the one of Problem (B), although it is not necessary for proving the sliding mode result.

Theorem 2.9 (Further regularity). Assume the same hypotheses as in Theorem 2.7 and the condition (2.24). Then the components $\varphi$ and $\xi$ of a solution $(w, \varphi, \xi, \sigma)$ given by Theorem 2.7] satisfy

$$
\begin{gathered}
\varphi \in W^{1, \infty}(0, T ; H) \cap H^{1}(0, T ; V) \cap L^{\infty}(0, T ; W), \\
\xi \in L^{\infty}(0, T ; H)
\end{gathered}
$$

and

$$
\|\varphi\|_{W^{1, \infty}(0, T ; H) \cap H^{1}(0, T ; V) \cap L^{\infty}(0, T ; W)}+\|\xi\|_{L^{\infty}(0, T ; H)} \leq C_{9}(1+\rho),
$$

where $C_{9}>0$ is a constant independent of $\rho$.

Theorem 2.10 (Sliding mode). Assume (2.1) (2.5) and (2.21). Then there exist $\rho^{*}>0$, such that the following condition is fulfilled: for every $\rho>\rho^{*}$ and for every solution $(w, \varphi, \xi, \sigma)$ to the problem (2.16) (2.20) there exists a time $T^{*} \in[0, T)$, such that

$$
\varphi(t)=\varphi^{*}, \quad \text { a.e. in } \Omega, \text { for a.a. } t \in\left(T^{*}, T\right) .
$$

Remark 2.11. We can make a remark similar to Remark 2.6. In this case we define

$$
\begin{aligned}
& C_{10}=\gamma C_{6}+\left\|\beta\left(\varphi^{*}\right)\right\|_{H}+\operatorname{Lip}(\pi) C_{6}+\pi(0)(T|\Omega|)^{1 / 2}+\left\|\Delta \varphi^{*}\right\|_{H}, \\
& \psi_{0}=\left\|\varphi_{0}-\varphi^{*}\right\|_{H},
\end{aligned}
$$

where the constant $C_{6}$ is given by Theorem 2.7 and $\operatorname{Lip}(\pi)$ denotes the Lipschitz constant of $\pi$. We may choose

$$
\rho^{*}=\frac{\psi_{0}}{T}+C_{10}
$$

and we have the following bound for $T^{*}$ :

$$
T^{*} \leq \frac{\psi_{0}}{\rho-C_{10}}<T .
$$

We recall for the reader's convenience a slightly modified version of the Young inequality. For $a, b, r \in$ $\mathbb{R}$ and $a, b, r>0$ it holds true that

$$
a b \leq \frac{1}{2 r} a^{2}+\frac{r}{2} b^{2} .
$$


We refer to the equation above as the Young inequality and it will be widely used throughout this work. For simplicity, we will often omit to write $\mathrm{d} x, \mathrm{~d} s$, etc., in integrals. In order to keep the formulae clear and to avoid boring calculations, we will use the symbol $C$ to denote a large-enough constant. This means that $C$ may change from line to line and even in the same chain of inequalities, and that its value is chosen to satisfy the inequality where it appears. While $C$ may depend only on the data of the problem, e.g. $\Omega, T$ and the initial data, $C$ is always independent of $\rho$ and the later-introduced parameter $\varepsilon$. In the proofs of continuous dependence of the solutions, $C$ will be also independent of the data corresponding to the single solutions.

\section{$3 \quad$ Existence proofs}

The proofs of the existence results in Theorems 2.1 and 2.7 go along the following line. First of all, we will introduce the Yosida approximations of Sign and $\beta$. In the following subsection, we will make use of the Faedo-Galerkin method, in order to approximate the solutions. Then we will make certain a priori estimates that give uniform bounds on the approximate solutions. Finally we will take the limit of the approximate solutions and we will prove that the limit is actually a solution to the problem.

\subsection{Yosida approximations}

In this subsection we recall a few facts regarding the theory of the Yosida approximation of maximal monotone operators and the Moreau-Yosida regularization of convex functions (see e.g. 4, Ch. 2] or [1, Ch. 2] for an introduction to Yosida approximation; see [13, Ch. 15] for Yosida regularization in metric spaces). After considering the abstract case, we will soon apply the results to the functions $\widehat{\beta}$ and $\|\cdot\|_{H}$.

We start with the definition of Moreau-Yosida regularization. Given a Hilbert space $X$ (whose norm is denoted by $\|\cdot\|)$, a proper, convex, l.s.c. function $\Phi: X \rightarrow[0,+\infty]$, and $\varepsilon>0$, we define the MoreauYosida regularization $\Phi_{\varepsilon}$ as

$$
\Phi_{\varepsilon}(v)=\inf _{w \in X}\left\{\frac{1}{2 \varepsilon}\|v-w\|^{2}+\Phi(w)\right\} .
$$

We incidentally notice that the infimum in the definition above is attained. The following proposition summarizes the properties, which will be used later on, of the Moreau-Yosida regularization.

Proposition 3.1. Let $\Phi: X \rightarrow[0,+\infty]$ be a convex, proper, l.s.c. function. Then, the following conclusions hold

1) $\Phi_{\varepsilon}$ is convex and continuous;

2) $\Phi_{\varepsilon}(v)<+\infty$ and $\Phi_{\varepsilon}(v) \leq \Phi(x)$ for all $v \in X$;

3) $\Phi_{\varepsilon}(v)$ converges monotonically to $\Phi(v)$ as $\varepsilon \rightarrow 0$;

4) $\liminf _{\varepsilon \rightarrow 0} \Phi_{\varepsilon}\left(v_{\varepsilon}\right) \geq \Phi(v)$, if $v_{\varepsilon}$ is a sequence converging to $v$;

5) $\Phi_{\varepsilon}$ is Fréchet-differentiable, the differential $\partial \Phi_{\varepsilon}$ is $\varepsilon^{-1}$-Lipschitz-continuous, and

$$
\left\|\partial \Phi_{\varepsilon}(x)\right\| \leq\|y\| \quad \forall x \in X, y \in \partial \Phi(x) .
$$

The differential $\partial \Phi_{\varepsilon}$ coincides with the Yosida approximation of the maximal monotone operator $\partial \Phi$.

At this point, we introduce $\widehat{\beta}_{\varepsilon}: \mathbb{R} \rightarrow \mathbb{R}$ and $\beta_{\varepsilon}=\partial \widehat{\beta}_{\varepsilon}: \mathbb{R} \rightarrow \mathbb{R}$, the Moreau-Yosida regularization of $\widehat{\beta}$ and Yosida approximation of $\beta$, respectively. It follows immediately from the previous proposition and the fact that $\widehat{\beta}(0)=0$ and $\beta(0) \ni 0$, that

$$
\begin{aligned}
& \beta_{\varepsilon}(0)=0, \quad \widehat{\beta}_{\varepsilon}(0)=0, \\
& \left|\beta_{\varepsilon}(r)-\beta_{\varepsilon}(s)\right| \leq \frac{1}{\varepsilon}|r-s|, \quad 0 \leq \widehat{\beta}_{\varepsilon}(r) \leq \frac{1}{2 \varepsilon} r^{2}, \\
& \left|\beta_{\varepsilon}(r)\right| \leq\left|\beta^{\circ}(r)\right|, \quad \widehat{\beta}_{\varepsilon}(r) \leq \widehat{\beta}(r),
\end{aligned}
$$

for all $t, s \in \mathbb{R}$, where $\beta^{\circ}(r)$ denotes the element of $\beta(r)$ having minimum modulus.

In the same way, we introduce the Moreau-Yosida regularization $\|\cdot\|_{H, \varepsilon}: H \rightarrow \mathbb{R}$ and the Yosida approximation $\operatorname{Sign}_{\varepsilon}: H \rightarrow H$. It holds that

$$
\|v\|_{H, \varepsilon}:=\min _{w \in H}\left\{\frac{1}{2 \varepsilon}\|v-w\|_{H}^{2}+\|w\|_{H}\right\}= \begin{cases}\|v\|_{H}-\frac{\varepsilon}{2} & \text { if }\|v\|_{H} \geq \varepsilon, \\ \frac{\|v\|_{H}^{2}}{2 \varepsilon} & \text { if }\|v\|_{H} \leq \varepsilon .\end{cases}
$$


Indeed, if we differentiate the convex function $w \mapsto \frac{1}{2 \varepsilon}\|v-w\|_{H}^{2}+\|w\|_{H}$, we obtain

$$
w+\varepsilon \operatorname{Sign} w \ni v
$$

yielding

$$
w= \begin{cases}\left(1-\frac{\varepsilon}{\|v\|_{H}}\right) v & \text { if }\|v\|_{H} \geq \varepsilon \\ 0 & \text { if }\|v\|_{H} \leq \varepsilon\end{cases}
$$

thus we can substitute $w$ in the minimum of equation 3.5. We calculate the Yosida approximation of Sign, by differentiating (3.5), obtaining

$$
\operatorname{Sign}_{\varepsilon}(v)=\frac{v}{\max \left\{\varepsilon,\|v\|_{H}\right\}}= \begin{cases}\frac{v}{\|v\|_{H}} & \text { if }\|v\|_{H} \geq \varepsilon \\ \frac{v}{\varepsilon} & \text { if }\|v\|_{H} \leq \varepsilon\end{cases}
$$

which imply

$$
\left(\operatorname{Sign}_{\varepsilon}(v), v\right) \geq\|v\|_{H, \varepsilon} .
$$

Finally, we point out that the Moreau-Yosida regularization converges uniformly in $H$, i.e.

$$
\sup \left\{\|v\|_{H}-\|v\|_{H, \varepsilon}: v \in H\right\} \leq \frac{\varepsilon}{2}
$$

\subsection{Existence of solutions for Problem (A)}

Faedo-Galerkin approximation. In order to use the Faedo-Galerkin method, we need to introduce a few notations. We take $\left\{v_{i}\right\}_{i=1}^{+\infty}$ a complete orthogonal set of $V$ given by the eigenfunctions of the Laplace operator coupled with Neumann conditions, i.e.

$$
-\triangle v_{i}=\lambda_{i} v_{i} \text { on } \Omega, \quad \partial_{n} v_{i}=0 \text { on } \Gamma
$$

where $\lambda_{i} \leq \lambda_{i+1}, i \in \mathbb{N}$, are the eigenvalues of the Laplace operator. We define $V_{n}:=\operatorname{span}\left\{v_{1}, \ldots, v_{n}\right\}$ and let $P_{n}: V \rightarrow V$ be the orthogonal projector on $V_{n}$. We know that $\cup_{i=1}^{+\infty} V_{n}$ is dense in $V$. It is still true that $\left\{v_{i}\right\}_{i=1}^{+\infty}$ is a complete orthogonal set for $H$ and $W$. Moreover, the operator $P_{n}$ can be extended or restricted to $H$ and $W$ respectively and the extension and the restriction are still orthogonal projectors in the spaces $H$ and $W$. We recall that if $v \in X$ then

$$
P_{n}(v) \rightarrow v \text { strongly in } X \quad \text { and } \quad\left\|P_{n}(v)\right\|_{X} \leq\|v\|_{X}
$$

where $X$ can be either $H, V$ or $W$. Using standard density results, we take $f_{n} \in C^{0}([0, T] ; H)$, such that $f_{n}$ converges strongly to $f$ in $L^{2}(0, T ; H)$. We now project the initial data as well as the target function $\eta^{*}$ :

$$
\begin{aligned}
& \vartheta_{0, n}:=P_{n} \vartheta_{0}, \quad w_{0, n}:=P_{n} w_{0}, \quad \varphi_{0, n}:=P_{n} \varphi_{0}, \\
& \eta_{n}^{*}:=P_{n} \eta^{*} .
\end{aligned}
$$

The new problem is now to find two functions $w_{n} \in C^{2}\left([0, T] ; V_{n}\right)$ and $\varphi_{n} \in C^{1}\left([0, T] ; V_{n}\right)$, such that

$$
\begin{aligned}
& \left(\partial_{t}^{2} w_{n}+l \partial_{t} \varphi_{n}-\kappa \Delta \partial_{t} w_{n}-\tau \Delta w_{n}\right. \\
& \left.+\rho \operatorname{Sign}_{\varepsilon}\left(\partial_{t} w_{n}+\alpha \varphi_{n}-\eta_{n}^{*}\right), v\right)=\left(f_{n}, v\right), \quad \forall v \in V_{n}, \text { in }[0, T], \\
& \left(\partial_{t} \varphi_{n}-\triangle \varphi_{n}+\beta_{\varepsilon}\left(\varphi_{n}\right)+\pi\left(\varphi_{n}\right), v\right)=\gamma\left(\partial_{t} w_{n}, v\right), \quad \forall v \in V_{n}, \text { in }[0, T], \\
& \partial_{t} w_{n}(0)=\vartheta_{0, n}, \quad w_{n}(0)=w_{n, 0}, \quad \varphi_{n}(0)=\varphi_{n, 0} .
\end{aligned}
$$

This is a non-linear system of ordinary differential equations of the second and first order in the variables $w_{n}$ and $\varphi_{n}$ respectively. The non-linearity is only given by $\operatorname{Sign}_{\varepsilon}, \beta_{\varepsilon}$, and $\pi$, which are all Lipschitzcontinuous functions. Hence, by Cauchy-Lipschitz theorem, there exists a unique solution $\left(w_{n}, \varphi_{n}\right)$ defined on $[0, T]$. 
First a priori estimate. We test equation (3.11) and equation (3.12) by taking $v=\partial_{t} w_{n}+\alpha \varphi_{n}-\eta_{n}^{*}$ and $v=\partial_{t} \varphi_{n}$. We sum with $\frac{1}{2} \frac{\mathrm{d}}{\mathrm{d} t}\left\|\varphi_{n}\right\|_{H}^{2}-\left(\varphi_{n}, \partial_{t} \varphi_{n}\right)=0$ obtaining

$$
\begin{aligned}
& \frac{1}{2} \frac{\mathrm{d}}{\mathrm{d} t}\left\|\partial_{t} w_{n}\right\|_{H}^{2}+\left(\partial_{t}^{2} w_{n}, \alpha \varphi_{n}-\eta_{n}^{*}\right)+l\left(\partial_{t} \varphi_{n}, \partial_{t} w_{n}+\alpha \varphi_{n}-\eta_{n}^{*}\right) \\
& +\kappa\left\|\partial_{t} \nabla w_{n}\right\|_{H}^{2}+\kappa\left(\partial_{t} \nabla w_{n}, \nabla\left(\alpha \varphi_{n}-\eta_{n}^{*}\right)\right) \\
& +\frac{\tau}{2} \frac{\mathrm{d}}{\mathrm{d} t}\left\|\nabla w_{n}\right\|_{H}^{2}+\tau\left(\nabla w_{n}, \nabla\left(\alpha \varphi_{n}-\eta_{n}^{*}\right)\right) \\
& +\rho\left(\operatorname{Sign}_{\varepsilon}\left(\partial_{t} w_{n}+\alpha \varphi_{n}-\eta_{n}^{*}\right), \partial_{t} w_{n}+\alpha \varphi_{n}-\eta_{n}^{*}\right) \\
& +\left\|\partial_{t} \varphi_{n}\right\|_{H}^{2}+\frac{1}{2} \frac{\mathrm{d}}{\mathrm{d} t}\left(\left\|\varphi_{n}\right\|_{H}^{2}+\left\|\nabla \varphi_{n}\right\|_{H}^{2}\right) \\
& +\frac{\mathrm{d}}{\mathrm{d} t} \int_{\Omega} \widehat{\beta}_{\varepsilon}\left(\varphi_{n}\right)+\left(\left(\pi\left(\varphi_{n}\right)-\varphi_{n}\right), \partial_{t} \varphi_{n}\right) \\
& =\left(f_{n}(t), \partial_{t} w_{n}+\alpha \varphi_{n}-\eta_{n}^{*}\right)+\gamma\left(\partial w_{n}, \partial_{t} \varphi_{n}\right) .
\end{aligned}
$$

We integrate between 0 and $t$, and, recalling that $\left(\operatorname{Sign}_{\varepsilon}(v), v\right) \geq\|v\|_{H, \varepsilon}$, we get

$$
\begin{aligned}
& \frac{1}{2}\left\|\partial_{t} w_{n}(t)\right\|_{H}^{2}+\kappa \int_{0}^{t}\left\|\partial_{t} \nabla w_{n}\right\|_{H}^{2}+\frac{\tau}{2}\left\|\nabla w_{n}(t)\right\|_{H}^{2} \\
& +\rho \int_{0}^{t}\left\|\partial_{t} w_{n}+\alpha \varphi_{n}+\eta_{n}^{*}\right\|_{H, \varepsilon}+\int_{0}^{t}\left\|\partial_{t} \varphi_{n}\right\|_{H}^{2} \\
& +\frac{1}{2}\left\|\varphi_{n}(t)\right\|_{V}^{2}+\int_{\Omega} \widehat{\beta}_{\varepsilon}\left(\varphi_{n}(t)\right) \\
& \leq \frac{1}{2}\left\|\vartheta_{0, n}\right\|_{H}^{2}+\frac{\tau}{2}\left\|\nabla w_{0, n}\right\|_{H}^{2}+\frac{1}{2}\left\|\varphi_{0, n}\right\|_{V}^{2}+\left\|\widehat{\beta}_{\varepsilon}\left(\varphi_{0, n}\right)\right\|_{L^{1}(\Omega)} \\
& +\int_{0}^{t}\left(\partial_{t} w_{n}, \alpha \partial_{t} \varphi_{n}\right)+\left(\vartheta_{0, n}, \alpha \varphi_{0, n}-\eta_{n}^{*}\right)-\left(\partial_{t} w_{n}(t), \alpha \varphi(t)-\eta_{n}^{*}\right) \\
& -l \int_{0}^{t}\left(\partial_{t} \varphi_{n}, \partial_{t} w_{n}+\alpha \varphi_{n}-\eta_{n}^{*}\right)-\kappa \int_{0}^{t}\left(\partial_{t} \nabla w_{n}, \nabla\left(\alpha \varphi_{n}-\eta_{n}^{*}\right)\right) \\
& -\tau \int_{0}^{t}\left(\nabla w_{n}, \nabla\left(\alpha \varphi_{n}-\eta_{n}^{*}\right)\right)-\int_{0}^{t}\left(\pi\left(\varphi_{n}\right)-\varphi_{n}, \partial_{t} \varphi_{n}\right) \\
& +\int_{0}^{t}\left(f_{n}, \partial_{t} w_{n}+\alpha \varphi_{n}-\eta_{n}^{*}\right)+\int_{0}^{t}\left(\partial_{t} w_{n}, \partial_{t} \varphi_{n}\right) .
\end{aligned}
$$

We need now to control the summands of the left side of (3.14). By (3.9) we have that

$$
\left\|\vartheta_{0, n}\right\|_{H}^{2} \leq\left\|\vartheta_{0}\right\|_{H}^{2} \leq C,
$$

and similarly we can control $\left\|\nabla w_{0, n}\right\|_{H}$ and $\left\|\varphi_{0, n}\right\|_{V}$. For the last initial datum we note

$$
\int_{\Omega} \widehat{\beta}_{\varepsilon}\left(\varphi_{n}\right) \leq \int_{\Omega} \frac{1}{2 \varepsilon}\left|\varphi_{0, n}\right|^{2} \leq \frac{1}{2 \varepsilon}\left\|\varphi_{0}\right\|_{H}^{2} .
$$

Using the Young inequality (2.45) we find

$$
\int_{0}^{t}\left(\partial_{t} w_{n}, \alpha \partial_{t} \varphi_{n}\right) \leq \frac{\alpha^{2}}{2} \int_{0}^{t}\left\|\partial_{t} w_{n}\right\|_{H}^{2}+\frac{1}{2} \int_{0}^{t}\left\|\partial_{t} \varphi_{n}\right\|_{H}^{2} .
$$

The next step is easier as

$$
\left(\vartheta_{0, n}, \alpha \varphi_{0, n}-\eta_{n}^{*}\right) \leq\left\|\vartheta_{0}\right\|_{H}^{2}+\frac{\alpha^{2}}{2}\left\|\varphi_{0}\right\|_{H}^{2}+\frac{1}{2}\left\|\eta^{*}\right\|_{H}^{2} \leq C .
$$

Again, owing to the Young inequality, we infer

$$
\begin{aligned}
-\left(\partial_{t} w_{n}(t), \alpha \varphi_{n}(t)-\eta_{n}^{*}\right) & \leq \frac{1}{4}\left\|\partial_{t} w_{n}(t)\right\|_{H}^{2}+\left\|\alpha \varphi_{n}(t)-\eta_{n}^{*}\right\|_{H}^{2} \\
& \leq \frac{1}{4}\left\|\partial_{t} w_{n}(t)\right\|_{H}^{2}+2 \alpha^{2}\left\|\varphi_{n}(t)\right\|_{H}^{2}+C .
\end{aligned}
$$


Since

$$
\begin{aligned}
\left\|\varphi_{n}(t)\right\|_{H}^{2} & =\left\|\varphi_{0, n}\right\|_{H}^{2}+2 \int_{0}^{t}\left(\varphi_{n}, \partial_{t} \varphi_{n}\right) \\
& \leq\left\|\varphi_{0}\right\|_{H}^{2}+8 \alpha^{2} \int_{0}^{t}\left\|\varphi_{n}\right\|_{H}^{2}+\frac{1}{8 \alpha^{2}} \int_{0}^{t}\left\|\partial_{t} \varphi_{n}\right\|_{H}^{2},
\end{aligned}
$$

we find

$$
-\left(\partial_{t} w_{n}(t), \alpha \varphi_{n}(t)-\eta_{n}^{*}\right) \leq \frac{1}{4}\left\|\partial_{t} w_{n}(t)\right\|_{H}^{2}+\frac{1}{4} \int_{0}^{t}\left\|\partial_{t} \varphi\right\|_{H}^{2}+C\left(1+\int_{0}^{t}\left\|\varphi_{n}\right\|_{H}^{2}\right) .
$$

Using the same technique we deduce

$$
\begin{gathered}
-l \int_{0}^{t}\left(\partial_{t} \varphi_{n}, \partial_{t} w_{n}+\alpha \varphi_{n}-\eta_{n}^{*}\right) \\
\leq \frac{1}{8} \int_{0}^{t}\left\|\partial_{t} \varphi_{n}\right\|_{H}^{2}+C\left(1+\int_{0}^{t}\left\|\partial_{t} w_{n}\right\|_{H}^{2}+\int_{0}^{t}\left\|\varphi_{n}\right\|_{H}^{2}\right) \\
-\kappa \int_{0}^{t}\left(\partial_{t} \nabla w_{n}, \nabla\left(\alpha \varphi_{n}-\eta_{n}^{*}\right) \leq \frac{\kappa}{2} \int_{0}^{t}\left\|\partial_{t} \nabla w_{n}\right\|_{H}^{2}+\alpha^{2} \int_{0}^{t}\left\|\varphi_{n}\right\|_{H}^{2}+C\right. \\
-\tau \int_{0}^{t}\left(\nabla w_{n}, \nabla\left(\alpha \varphi_{n}+\eta_{n}^{*}\right) \leq \frac{\tau}{2} \int_{0}^{t}\left\|\nabla w_{n}\right\|_{H}^{2}+\tau \alpha^{2} \int_{0}^{t}\left\|\nabla \varphi_{n}\right\|_{H}^{2}+C .\right.
\end{gathered}
$$

Then, recalling that $\pi$ is Lipschitz-continuous, we have

$$
\begin{aligned}
-\int_{0}^{t}\left(\pi\left(\varphi_{n}\right)-\varphi_{n}, \partial_{t} \varphi_{n}\right) & \leq 4 \int_{0}^{t}\left\|\pi\left(\varphi_{n}\right)-\varphi_{n}\right\|_{H}^{2}+\frac{1}{16} \int_{0}^{t}\left\|\partial_{t} \varphi_{n}\right\|_{H}^{2} \\
& \leq C\left(1+\int_{0}^{t}\left\|\varphi_{n}\right\|_{H}^{2}\right)+\frac{1}{16} \int_{0}^{t}\left\|\partial_{t} \varphi_{n}\right\|_{H}^{2} \\
\int_{0}^{t}\left(f_{n}, \partial_{t} w_{n}+\alpha \varphi_{n}-\eta_{n}^{*}\right) & \leq C\left(1+\int_{0}^{t}\left\|\partial_{t} w_{n}\right\|_{H}^{2}+\int_{0}^{t}\left\|\varphi_{n}\right\|_{H}^{2}\right) \\
\int_{0}^{t}\left(\partial_{t} w_{n}, \partial_{t} \varphi_{n}\right) & \leq 8 \int_{0}^{t}\left\|\partial_{t} w_{n}\right\|_{H}^{2}+\frac{1}{32} \int_{0}^{t}\left\|\partial_{t} \varphi_{n}\right\|_{H}^{2} .
\end{aligned}
$$

We put everything together, obtaining

$$
\begin{aligned}
& \frac{1}{32}\left\|\partial_{t} w_{n}(t)\right\|_{H}^{2}+\frac{\kappa}{2} \int_{0}^{t}\left\|\partial_{t} \nabla w_{n}\right\|_{H}^{2}+\frac{\tau}{2}\left\|\nabla w_{n}(t)\right\|_{H}^{2} \\
& +\rho \int_{0}^{t}\left\|\partial_{t} w_{n}+\alpha \varphi_{n}+\eta_{n}^{*}\right\|_{H, \varepsilon}+\frac{1}{4} \int_{0}^{t}\left\|\partial_{t} \varphi_{n}\right\|_{H}^{2}+\frac{1}{2}\left\|\varphi_{n}(t)\right\|_{V}^{2} \\
& +\int_{\Omega} \widehat{\beta}_{\varepsilon}\left(\varphi_{n}(t)\right) \leq C\left(1+\varepsilon^{-1}+\int_{0}^{t}\left\|\partial_{t} w_{n}\right\|_{H}^{2}+\int_{0}^{t}\left\|\varphi_{n}\right\|_{V}^{2}+\int_{0}^{t}\left\|\nabla w_{n}\right\|_{H}^{2} \cdot\right)
\end{aligned}
$$

We use now the Gronwall lemma deducing

$$
\begin{aligned}
& \left\|w_{n}\right\|_{W^{1, \infty}(0, T ; H) \cap H^{1}(0, T ; V)}+\left\|\varphi_{n}\right\|_{H^{1}(0, T ; H) \cap L^{\infty}(0, T ; V)} \\
& +\rho \int_{0}^{T}\left\|\partial_{t} w_{n}+\alpha \varphi_{n}+\eta_{n}^{*}\right\|_{H, \varepsilon}+\left\|\widehat{\beta}_{\varepsilon}\left(\varphi_{n}\right)\right\|_{L^{\infty}\left(0, T ; L^{1}(\Omega)\right)} \leq C\left(1+\varepsilon^{-1}\right) .
\end{aligned}
$$

Remark 3.2. Unfortunately, we are not able to provide an estimate independent of $\varepsilon$, preventing the possibility of taking the limit as $\varepsilon \rightarrow 0$. For the moment, we do not worry about this trouble since our first aim is to take the limit as $n \rightarrow \infty$.

We stress that the only dependence on $\varepsilon$ in (3.16) arises in (3.15). We anticipate that the other estimates have a dependence on $\varepsilon$ because we will use this estimate to prove them. Hence, when we will be able to fine-tune equation (3.15) removing the dependence on $\varepsilon$, all estimates will work perfectly, being independent of $\varepsilon$.

Finally, we point out that the term $C\left(1+\varepsilon^{-1}\right)$ could be slightly refined in $C\left(1+\varepsilon^{-1 / 2}\right)$. We will be sloppy in carrying out the dependence on $\varepsilon$ because, as we have just said, we will remove this dependence, and, at this stage, we only want estimates independent of $n$. 
Second a priori estimate. We define $g_{1}:[0, T] \rightarrow H$ as $g_{1}(t)=\gamma \partial_{t} w_{n}(t)-\partial_{t} \varphi_{n}(t)-\pi\left(\varphi_{n}(t)\right)$. Due to the first a priori estimate and the Lipschitz-continuity of $\pi$, we have that

$$
\left\|g_{1}\right\|_{L^{2}(0, T ; H)} \leq C\left(1+\varepsilon^{-1}\right) .
$$

We rewrite (3.12) as

$$
-\left(\triangle \varphi_{n}(t), v\right)+\left(\beta_{\varepsilon}\left(\varphi_{n}(t)\right), v\right)=\left(g_{1}(t), v\right),
$$

and we test with $v=-\triangle \varphi_{n}(t)$ :

$$
\left\|\triangle \varphi_{n}(t)\right\|_{H}^{2}-\left(\beta_{\varepsilon}\left(\varphi_{n}(t)\right), \triangle \varphi_{n}(t)\right)=-\left(g_{1}, \triangle \varphi_{n}\right) \leq\left\|g_{1}(t)\right\|_{H}\left\|\triangle \varphi_{n}(t)\right\|_{H} .
$$

The second term is positive, because of

$$
-\left(\beta_{\varepsilon}\left(\varphi_{n}\right), \triangle \varphi_{n}\right)=-\int_{\Omega} \beta_{\varepsilon}\left(\varphi_{n}\right) \triangle \varphi_{n}=\int_{\Omega} \beta_{\varepsilon}^{\prime}\left(\varphi_{n}\right)\left|\nabla \varphi_{n}\right|^{2} \geq 0,
$$

then yielding $\left\|\triangle \varphi_{n}(t)\right\|_{H} \leq\left\|g_{1}(t)\right\|_{H}$. Since $P_{n}\left(\beta_{\varepsilon}\left(\varphi_{n}\right)\right)=P_{n}\left(g_{1}\right)+\triangle \varphi_{n}$, owing to elliptic regularity, we conclude that

$$
\left\|\varphi_{n}\right\|_{L^{2}(0, T ; W)}+\left\|P_{n}\left(\beta_{\varepsilon}\left(\varphi_{n}\right)\right)\right\|_{L^{2}(0, T ; H)} \leq C\left(1+\varepsilon^{-1}\right) .
$$

Third a priori estimate. We define $\eta, g_{2}:[0, T] \rightarrow V_{n}$ as

$$
\begin{gathered}
\eta(t):=\partial_{t} w_{n}(t)+\alpha \varphi_{n}(t)-\eta_{n}^{*} \\
g_{2}(t):=(\alpha-l) \partial_{t} \varphi_{n}(t)-\alpha \kappa \Delta \varphi_{n}(t)+\kappa \triangle \eta_{n}^{*} \\
+\tau \triangle\left(w_{0, n}+\alpha \int_{0}^{t} \varphi_{n}(s) \mathrm{d} s+t \eta_{n}^{*}\right)+f_{n}(t)
\end{gathered}
$$

for $t \in[0, T]$. Thanks to equations (3.16) and (3.17) we have that

$$
\left\|g_{2}\right\|_{L^{2}(0, T ; H)}+\|\eta\|_{L^{2}(0, T ; H)} \leq C\left(1+\varepsilon^{-1}\right) .
$$

Moreover, equation (2.5) implies

$$
\|\eta(0)\|_{V}=\left\|\vartheta_{0, n}+\alpha \varphi_{0, n}+\eta_{n}^{*}\right\|_{V} \leq C .
$$

We rewrite equation (3.11) as

$$
\left(\partial_{t} \eta-\kappa \Delta \eta-\tau \int_{0}^{t} \triangle \eta(s) \mathrm{d} s+\rho \operatorname{Sign}_{\varepsilon}(\eta), v\right)=\left(g_{2}, v\right) .
$$

In view of equation (3.5), it is clear that

$$
\begin{aligned}
\left(\triangle \eta(t), \int_{0}^{t} \triangle \eta(s) \mathrm{d} s\right) & =\frac{1}{2} \frac{\mathrm{d}}{\mathrm{d} t}\left\|\int_{0}^{t} \Delta \eta(s) \mathrm{d} s\right\|_{H}^{2}, \\
-\int_{\Omega} \operatorname{Sign}_{\varepsilon}(\eta) \triangle \eta & =\int_{\Omega} \nabla \operatorname{Sign}_{\varepsilon}(\eta) \cdot \nabla \eta \geq 0 \\
-\int_{\Omega} \partial_{t} \eta \triangle \eta & =\frac{1}{2} \frac{\mathrm{d}}{\mathrm{d} t} \int_{\Omega}|\nabla \eta|^{2} .
\end{aligned}
$$

Thus, we test (3.2) with $v=-\Delta \eta(t)$ and we integrate over time finding

$$
\begin{aligned}
\frac{1}{2}\|\nabla \eta(t)\|_{H}^{2} & +\kappa \int_{0}^{t}\|\Delta \eta\|_{H}^{2}+\frac{\tau}{2}\left\|\int_{0}^{t} \Delta \eta \mathrm{d} s\right\|_{H}^{2} \\
& \leq \frac{1}{2}\|\nabla \eta(0)\|_{H}^{2}-\int_{0}^{t}\left(g_{2}, \triangle \eta\right) \\
& \leq C+\frac{\kappa}{2} \int_{0}^{t}\|\Delta \eta\|_{H}^{2}+\frac{1}{2 \kappa} \int_{0}^{t}\left\|g_{2}\right\|_{H}^{2} .
\end{aligned}
$$


Hence, we infer

$$
\|\nabla \eta\|_{L^{\infty}(0, T ; H)}+\|\triangle \eta\|_{L^{2}(0, T ; H)} \leq C\left(1+\varepsilon^{-1}\right),
$$

which, together with elliptic regularity, implies $\|\eta\|_{L^{2}(0, T ; W)} \leq C\left(1+\varepsilon^{-1}\right)$. Thus, it turns out that

$$
\left\|\partial_{t} w_{n}\right\|_{L^{\infty}(0, T ; V)}+\left\|\partial_{t} w_{n}\right\|_{L^{2}(0, T ; W)} \leq C\left(1+\varepsilon^{-1}\right) .
$$

Finally, as $\left\|w_{n}\right\|_{L^{\infty}(0, T ; H)} \leq C\left(1+\varepsilon^{-1}\right)$ and $w_{0, n} \in W$, we conclude that

$$
\left\|w_{n}\right\|_{W^{1, \infty}(0, T ; V) \cap H^{1}(0, T ; W)} \leq C\left(1+\varepsilon^{-1}\right) .
$$

Fourth a priori estimate. We define $g_{3}:[0, T] \rightarrow V_{n}$ as

$$
g_{3}(t)=g_{2}(t)+\kappa \Delta \eta(t)+\tau \int_{0}^{t} \triangle \eta(s) \mathrm{d} s, \quad t \in[0, T] .
$$

Again, it holds true that $\left\|g_{3}\right\|_{L^{2}(0, T ; H)} \leq C\left(1+\varepsilon^{-1}\right)$ by comparison. We rewrite equation (3.2) as

$$
\left(\eta_{t}+\rho \operatorname{Sign}_{\varepsilon}(\eta), v\right)=\left(g_{3}, v\right) .
$$

Since $\frac{\mathrm{d}}{\mathrm{d} t}\|\eta\|_{H, \varepsilon}=\left(\operatorname{Sign}_{\varepsilon}(\eta), \partial_{t} \eta\right)_{H}$, we can test (3.19) with $v=\partial_{t} \eta$ obtaining

$$
\begin{aligned}
\int_{0}^{t}\left\|\partial_{t} \eta\right\|_{H}^{2}+\rho\|\eta(t)\|_{H} & =\rho\|\eta(0)\|_{H}+\int_{0}^{t}\left(g_{3}(s), \partial_{t} \eta(s)\right) \mathrm{d} s \\
& \leq \rho C+\frac{1}{2} \int_{0}^{t}\left\|\partial_{t} \eta\right\|_{H}^{2}+\frac{1}{2} \int_{0}^{t}\left\|g_{3}\right\|_{H}^{2}
\end{aligned}
$$

Thus, $\left\|\partial_{t} \eta\right\|_{L^{2}(0, T ; H)}^{2} \leq C\left(1+\rho+\varepsilon^{-1}\right)$ and then, by comparison, we have that

$$
\left\|w_{n}\right\|_{H^{2}(0, T ; H)} \leq C\left(1+\rho^{1 / 2}+\varepsilon^{-1}\right) .
$$

Passage to the limit in the Faedo-Galerkin scheme. Making use of standard weak or weak* compactness results, possibly taking a subsequence, we have that $\left(w_{n}, \varphi_{n}\right)$ converges in the following topologies

$$
\begin{aligned}
w_{n} \rightarrow w_{\varepsilon} & \text { weakly in } H^{2}(0, T ; H) \cap H^{1}(0, T ; W), \\
\varphi_{n} \rightarrow \varphi_{\varepsilon} & \text { weakly in } H^{1}(0, T ; H) \cap L^{2}(0, T ; W), \\
w_{n} \rightarrow w_{\varepsilon} & \text { weakly* in } W^{1, \infty}(0, T ; V), \\
\varphi_{n} \rightarrow \varphi_{\varepsilon} & \text { weakly* in } L^{\infty}(0, T ; V),
\end{aligned}
$$

for a suitable pair $\left(w_{\varepsilon}, \varphi_{\varepsilon}\right)$. This implies, together with the generalized Ascoli theorem and the AubinLions theorem [28, Sec. 8, Cor. 4], the following strong convergences

$$
\begin{array}{ll}
w_{n} \rightarrow w_{\varepsilon} & \text { in } H^{1}(0, T ; V) \cap C^{1}([0, T] ; H), \\
\varphi_{n} \rightarrow \varphi_{\varepsilon} & \text { in } C^{0}([0, T] ; H) \cap L^{2}(0, T ; V) .
\end{array}
$$

Hence, we have the convergences

$$
\begin{aligned}
\operatorname{Sign}_{\varepsilon}\left(\partial_{t} w_{n}+\alpha \varphi_{n}-\eta_{n}^{*}\right) & \rightarrow \operatorname{Sign}_{\varepsilon}\left(\partial_{t} w_{\varepsilon}+\alpha \varphi_{\varepsilon}-\eta^{*}\right), \\
\pi\left(\varphi_{n}\right) & \rightarrow \pi\left(\varphi_{\varepsilon}\right) \\
\beta_{\varepsilon}\left(\varphi_{n}\right) & \rightarrow \beta_{\varepsilon}\left(\varphi_{\varepsilon}\right)
\end{aligned}
$$

in $C^{0}([0, T] ; H)$. We note that $\eta_{n}^{*} \rightarrow \eta^{*}$ and that the initial conditions hold true

$$
\partial_{t} w_{\varepsilon}(0)=\vartheta_{0}, \quad w_{\varepsilon}(0)=w_{0}, \quad \varphi_{\varepsilon}(0)=\varphi_{0}
$$

Indeed, the property (3.9) implies the strong convergence of the initial data and the target function $\eta^{*}$. We take $n, h \in \mathbb{N}$ with $n>h$ and $v \in V_{h} \subset V_{n}$. Since all the involved terms converge, we take the limit as $n \rightarrow+\infty$ in equations (3.11) and (3.12), obtaining

$$
\begin{aligned}
& \left(\partial_{t}^{2} w_{\varepsilon}+l \partial_{t} \varphi_{\varepsilon}-\kappa \Delta \partial_{t} w_{\varepsilon}-\tau \Delta w_{\varepsilon}\right. \\
& \left.+\rho \operatorname{Sign}_{\varepsilon}\left(\partial_{t} w_{\varepsilon}+\alpha \varphi_{\varepsilon}-\eta^{*}\right), v\right)=(f, v) \quad \forall v \in V_{h}, \text { a.e. in }(0, T), \\
& \left(\partial_{t} \varphi_{\varepsilon}-\triangle \varphi_{\varepsilon}+\beta_{\varepsilon}\left(\varphi_{\varepsilon}\right)+\pi\left(\varphi_{\varepsilon}\right), v\right)=\gamma\left(\partial_{t} w_{\varepsilon}, v\right) \quad \forall v \in V_{h}, \text { a.e. in }(0, T) .
\end{aligned}
$$


As $h$ is arbitrary, the above equations hold for all $v \in \cup_{h=1}^{+\infty} V_{h}$. By density of $\cup_{h=1}^{+\infty} V_{h}$ in $H$, we find

$$
\begin{aligned}
& \left(\partial_{t}^{2} w_{\varepsilon}+l \partial_{t} \varphi_{\varepsilon}-\kappa \Delta \partial_{t} w_{\varepsilon}-\tau \Delta w_{\varepsilon}\right. \\
& \left.+\rho \operatorname{Sign}_{\varepsilon}\left(\partial_{t} w_{\varepsilon}+\alpha \varphi_{\varepsilon}-\eta^{*}\right), v\right)=(f, v) \quad \forall v \in H, \text { a.e. in }(0, T), \\
& \left(\partial_{t} \varphi_{\varepsilon}-\triangle \varphi_{\varepsilon}+\beta_{\varepsilon}\left(\varphi_{\varepsilon}\right)+\pi\left(\varphi_{\varepsilon}\right), v\right)=\gamma\left(\partial_{t} w_{\varepsilon}, v\right) \quad \forall v \in H, \text { a.e. in }(0, T) .
\end{aligned}
$$

Passage to the limit as $\varepsilon \rightarrow 0$. Let $\xi_{\varepsilon}:=\beta_{\varepsilon}\left(\varphi_{\varepsilon}\right)$ and $\sigma_{\varepsilon}:=\operatorname{Sign}_{\varepsilon}\left(\partial_{t} w_{\varepsilon}+\alpha \varphi_{\varepsilon}-\eta^{*}\right)$. We now review the a priori estimates in order to remove the dependence on $\varepsilon$. All calculations are still working if we make the substitution

$$
\left(w_{\varepsilon}, \varphi_{\varepsilon}, \xi_{\varepsilon}, \sigma_{\varepsilon}\right) \longmapsto\left(w_{n}, \varphi_{n}, \beta_{\varepsilon}\left(\varphi_{n}\right), \operatorname{Sign}_{\varepsilon}\left(\partial_{t} w_{n}+\alpha \varphi_{n}-\eta_{n}^{*}\right)\right) .
$$

By Remark 3.2 the dependence on $\varepsilon$ is only given by equation (3.15). We observe that, owing to (3.4),

$$
\left\|\widehat{\beta}_{\varepsilon}\left(\varphi_{0}\right)\right\|_{L^{1}(\Omega)} \leq\left\|\widehat{\beta}\left(\varphi_{0}\right)\right\|_{L^{1}(\Omega)},
$$

and we had just made the first a priori estimate independent of $\varepsilon$ :

$$
\begin{aligned}
& \left\|w_{\varepsilon}\right\|_{W^{1, \infty}(0, T ; H) \cap H^{1}(0, T ; V)}+\left\|\varphi_{\varepsilon}\right\|_{H^{1}(0, T ; H) \cap L^{\infty}(0, T ; V)} \\
& +\rho \int_{0}^{T}\left\|\partial_{t} w_{\varepsilon}+\alpha \varphi_{\varepsilon}+\eta^{*}\right\|_{H, \varepsilon}+\left\|\widehat{\beta}_{\varepsilon}\left(\varphi_{\varepsilon}\right)\right\|_{L^{\infty}\left(0, T ; L^{1}(\Omega)\right)} \leq C .
\end{aligned}
$$

Having removed the dependence on $\varepsilon$ in the first estimate, all other estimates can be replicated obtaining

$$
\begin{aligned}
& \left\|\varphi_{\varepsilon}\right\|_{L^{2}(0, T ; W)}+\left\|\xi_{\varepsilon}\right\|_{L^{2}(0, T ; H)} \leq C, \\
& \left\|w_{\varepsilon}\right\|_{W^{1, \infty}(0, T ; V) \cap H^{1}(0, T ; W)} \leq C \\
& \left\|w_{\varepsilon}\right\|_{H^{2}(0, T ; H)} \leq C\left(1+\rho^{1 / 2}\right) .
\end{aligned}
$$

Moreover, because of the definition of the Sign operator, $\sigma_{\varepsilon}(t)$ is bounded, uniformly with respect to $t$ and $\varepsilon$, i.e.,

$$
\left\|\sigma_{\varepsilon}\right\|_{L^{\infty}(0, T ; H)} \leq 1
$$

We are now able to take the limit as $\varepsilon \rightarrow 0$ using the same compactness argument as before. There exists a quadruplet $(w, \varphi, \xi, \sigma)$ such that (a subsequence of) $\left(w_{\varepsilon}, \varphi_{\varepsilon}, \xi_{\varepsilon}, \sigma_{\varepsilon}\right)$ converges to $(w, \varphi, \xi, \sigma)$ in the same topologies as before. More precisely for $\partial_{t} w_{\varepsilon}+\alpha \varphi_{\varepsilon}, \xi_{\varepsilon}$, and $\sigma_{\varepsilon}$ we have that

$$
\begin{aligned}
\partial_{t} w_{\varepsilon}+\alpha \varphi_{\varepsilon} & \rightarrow \partial_{t} w+\alpha \varphi & & \text { in } C^{0}([0, T], H), \\
\xi_{\varepsilon} & \rightarrow \xi & & \text { weakly in } L^{2}(0, T ; H), \\
\sigma_{\varepsilon} & \rightarrow \sigma & & \text { weakly in } L^{2}(0, T ; H) .
\end{aligned}
$$

We take the limit in equation (3.27) and (3.28) obtaining (2.10) and (2.12), respectively. Since $\varphi_{\varepsilon}$ and $\partial_{t} w_{\varepsilon}$ converge strongly in $L^{2}(0, T ; H)=L^{2}(Q)$ and $\xi_{\varepsilon}$ and $\sigma_{\varepsilon}$ converge weakly, we deduce

$$
\begin{aligned}
\lim _{\varepsilon \rightarrow 0} \int_{Q} \xi_{\epsilon} \varphi_{\epsilon} & =\int_{Q} \xi \varphi, \\
\lim _{\varepsilon \rightarrow 0} \int_{Q} \sigma_{\epsilon}\left(\partial_{t} w_{\epsilon}+\alpha \varphi_{\epsilon}-\eta^{*}\right) & =\int_{Q} \sigma\left(\partial_{t} w+\alpha \varphi-\eta^{*}\right) .
\end{aligned}
$$

Hence, by [1, Prop. 2.2, p. 38] we have that

$$
\xi \in \beta(\varphi) \quad \text { and } \quad \sigma \in \operatorname{Sign}\left(\partial_{t} w+\alpha \varphi-\eta^{*}\right),
$$

almost everywhere, and the proof of the existence of the solutions is complete.

To conclude the proof of Theorem [2.1 we need to prove (2.22) and (2.23). Owing to the lower semicontinuity of the norms, the Fatou lemma, and part 4 of Proposition 3.1 we can take the inferior limit as $\varepsilon \rightarrow 0$ in (3.29)-(3.33) deducing

$$
\begin{aligned}
& \|w\|_{W^{1, \infty}(0, T ; V) \cap H^{1}(0, T ; W)}+\|\varphi\|_{H^{1}(0, T ; H) \cap L^{\infty}(0, T ; V) \cap L^{2}(0, T ; W)} \\
& +\rho\left\|w_{t}+\alpha \varphi-\eta^{*}\right\|_{L^{1}(0, T ; H)}+\|\widehat{\beta}(\varphi)\|_{L^{\infty}\left(0, T ; L^{1}(\Omega)\right)} \\
& +\|\xi\|_{L^{2}(0, T ; H)}+\|\sigma\|_{L^{\infty}(0, T ; H)} \leq C \\
& \|w\|_{H^{2}(0, T ; H)} \leq C\left(1+\rho^{1 / 2}\right) .
\end{aligned}
$$




\subsection{Existence of solutions for Problem (B)}

The proof of Theorem 2.7 is similar to the proof of the previous one. For this reason the steps with few relevance will be omitted.

Faedo-Galerkin approximation. Most of the first paragraph of the previous subsection can be replicated verbatim. The first difference appears in equation (3.10); in this case, we project the target function $\varphi^{*}$

$$
\varphi_{n}^{*}:=P_{n} \varphi^{*}
$$

The approximated problem is to find two functions $w_{n} \in C^{2}\left([0, T] ; V_{n}\right)$ and $\varphi_{n} \in C^{1}\left([0, T] ; V_{n}\right)$, such that

$$
\begin{aligned}
& \left(\partial_{t}^{2} w_{n}+l \partial_{t} \varphi_{n}-\kappa \Delta \partial_{t} w_{n}-\tau \Delta w_{n}, v\right)=\left(f_{n}, v\right) \\
& \quad \forall v \in V_{n}, \text { in }[0, T] \\
& \left(\partial_{t} \varphi_{n}-\triangle \varphi_{n}+\beta_{\varepsilon}\left(\varphi_{n}\right)+\pi\left(\varphi_{n}\right)+\rho \operatorname{Sign}_{\varepsilon}\left(\varphi_{n}-\varphi_{n}^{*}\right), v\right) \\
& \quad=\gamma\left(\partial_{t} w_{n}, v\right), \quad \forall v \in V_{n}, \quad \text { in }[0, T] \\
& \partial_{t} w_{n}(0)=\vartheta_{0, n}, \quad w_{n}(0)=w_{n, 0}, \quad \varphi_{n}(0)=\varphi_{n, 0} .
\end{aligned}
$$

Like for Problem (A), the system above has a unique solution, given by Cauchy-Lipschitz theorem.

First a priori estimate. We take $v=\partial_{t} w_{n}+l \varphi_{n}$ and $v=\kappa l^{2}\left(\varphi_{n}-\varphi_{n}^{*}\right)$ in equations (3.40) and (3.41), respectively. We sum up, integrate between 0 and $t$, and, taking (3.7) into account, reorder obtaining

$$
\begin{aligned}
& \frac{1}{2}\left\|\partial_{t} w_{n}+l \varphi_{n}\right\|_{H}^{2}+\kappa \int_{0}^{t}\left\|\partial_{t} \nabla w_{n}\right\|_{H}^{2}+\frac{\tau}{2}\left\|\nabla w_{n}\right\|_{H}^{2}+\kappa l \int_{0}^{t}\left(\partial_{t} \nabla w_{n}, \nabla \varphi_{n}\right) \\
& +\frac{\kappa l^{2}}{2}\left\|\varphi_{n}\right\|_{H}^{2}+\kappa l^{2} \int_{0}^{t}\left\|\nabla \varphi_{n}\right\|_{H}^{2}+\kappa l^{2} \int_{0}^{t}\left(\nabla \varphi_{n}, \nabla \varphi_{n}^{*}\right) \\
& +\kappa l^{2} \int_{0}^{t}\left(\beta_{\varepsilon}\left(\varphi_{n}\right)-\beta_{\varepsilon}\left(\varphi_{n}^{*}\right), \varphi_{n}-\varphi_{n}^{*}\right)+\rho \kappa l^{2} \int_{0}^{t}\left\|\varphi_{n}-\varphi_{n}^{*}\right\|_{H, \varepsilon} \\
& \leq \frac{1}{2}\left\|\partial_{t} w_{0, n}+l \varphi_{0, n}\right\|_{H}^{2}+\frac{\tau}{2}\left\|\nabla w_{0, n}\right\|_{H}^{2}+\frac{\kappa l^{2}}{2}\left\|\varphi_{0, n}\right\|_{H}^{2} \\
& -\tau l \int_{0}^{t}\left(\nabla w_{n}, \nabla \varphi_{n}\right)+\int_{0}^{t}\left(f_{n}, \partial_{t} w_{n}+l \varphi_{n}\right) \\
& -\kappa l^{2} \int_{0}^{t}\left(\pi\left(\varphi_{n}\right), \varphi_{n}-\varphi_{n}^{*}\right)+\gamma \kappa l^{2} \int_{0}^{t}\left(\partial_{t} w_{n}, \varphi_{n}-\varphi_{n}^{*}\right) \\
& -\kappa l^{2} \int_{0}^{t}\left(\beta_{\varepsilon}\left(\varphi_{n}^{*}\right), \varphi_{n}-\varphi_{n}^{*}\right)+\kappa l^{2}\left(\varphi_{n}, \varphi_{n}^{*}\right) .
\end{aligned}
$$

Regarding the left-hand side of the inequality above, using the Young inequality, it can be easily proven that

$$
\begin{aligned}
& \frac{1}{2}\left\|\partial_{t} w_{n}+l \varphi_{n}\right\|_{H}^{2}+\frac{\kappa l^{2}}{2}\left\|\varphi_{n}\right\|_{H}^{2} \geq \frac{l^{2} \kappa}{4}\left\|\varphi_{n}\right\|_{H}^{2}+\frac{\kappa}{2(\kappa+2)}\left\|\partial_{t} w_{n}\right\|_{H}^{2}, \\
& \left\|\partial_{t} \nabla w_{n}\right\|_{H}^{2}+l\left(\partial_{t} \nabla w_{n}, \nabla \varphi_{n}\right)+l^{2}\left\|\nabla \varphi_{n}\right\|_{H}^{2} \geq \frac{1}{2}\left(\left\|\partial_{t} \nabla w_{n}\right\|_{H}^{2}+l^{2}\left\|\nabla \varphi_{n}\right\|_{H}^{2}\right) .
\end{aligned}
$$

The non-constant terms in the right-hand side of the inequality are easily controlled via the Young inequality: we have that

$$
\begin{aligned}
& \int_{0}^{t}\left(\pi\left(\varphi_{n}\right), \varphi_{n}-\varphi_{n}^{*}\right) \leq C \int_{0}^{t}\left(\left\|\varphi_{n}\right\|_{H}^{2}+1\right) \\
& \tau l \int_{0}^{t}\left(\nabla w_{n}, \nabla \varphi_{n}\right) \leq \frac{\kappa l^{2}}{4} \int_{0}^{t}\left\|\nabla \varphi_{n}\right\|_{H}^{2}+\frac{\tau}{\kappa} \int_{0}^{t}\left\|\nabla w_{n}\right\|_{H}^{2}, \\
& \int_{0}^{t}\left(f_{n}, \partial_{t} w_{n}-l \varphi_{n}\right) \leq C+\int_{0}^{t}\left(\left\|\partial_{t} w_{n}\right\|_{H}^{2}+l^{2}\left\|\varphi_{n}\right\|_{H}^{2}\right), \\
& \int_{0}^{t}\left(\partial_{t} w_{n}, \varphi_{n}-\varphi_{n}^{*}\right), \leq C+\frac{1}{2} \int_{0}^{t}\left(\left\|\partial_{t} w_{n}\right\|_{H}^{2}+\left\|\varphi_{n}\right\|_{H}^{2}\right)
\end{aligned}
$$


as well as

$$
\begin{aligned}
& \kappa l^{2}\left(\varphi_{n}, \varphi_{n}^{*}\right) \leq \frac{\kappa l^{2}}{8}\left\|\varphi_{n}\right\|_{H}^{2}+2 \kappa l^{2}\left\|\varphi_{n}^{*}\right\|_{H}^{2}, \\
& \int_{0}^{t}\left(\beta_{\varepsilon}\left(\varphi_{n}^{*}\right), \varphi_{n}-\varphi_{n}^{*}\right) \leq \frac{1}{2} \int_{0}^{t}\left\|\beta_{\varepsilon}\left(\varphi_{n}^{*}\right)\right\|_{H}^{2}+\int_{0}^{t}\left\|\varphi_{n}\right\|_{H}^{2}+T\left\|\varphi_{n}^{*}\right\|_{H}^{2} .
\end{aligned}
$$

We notice that (cf. (3.3))

$$
\int_{0}^{t}\left\|\beta_{\varepsilon}\left(\varphi_{n}^{*}\right)\right\|_{H}^{2} \leq T \varepsilon^{-2}\left\|\varphi_{n}^{*}\right\|_{H}^{2} \leq C \varepsilon^{-2} .
$$

We can use Gronwall lemma obtaining

$$
\begin{aligned}
& \left\|w_{n}\right\|_{W^{1, \infty}(0, T ; H) \cap H^{1}(0, T ; V)}+\left\|\varphi_{n}\right\|_{L^{\infty}(0, T ; H) \cap L^{2}(0, T ; V)} \\
& +\rho \int_{0}^{T}\left\|\varphi_{n}-\varphi_{n}^{*}\right\|_{H, \varepsilon} \leq C\left(1+\varepsilon^{-1}\right) .
\end{aligned}
$$

The dependence on $\varepsilon$ is given only by equation (3.43). However, Remark 3.2 still remains valid, so the dependence on $\varepsilon$ in equation (3.44) will be later removed.

Second a priori estimate. We take $v=\partial_{t} \varphi_{n}$ in (3.41) and integrate over $(0, t)$, obtaining

$$
\begin{aligned}
& \int_{0}^{t}\left\|\partial_{t} \varphi_{n}\right\|_{H}^{2}+\left\|\nabla \varphi_{n}\right\|_{H}^{2}+\left\|\widehat{\beta}_{\varepsilon}\left(\varphi_{n}\right)\right\|_{L^{1}(\Omega)}+\rho\left\|\varphi_{n}-\varphi_{n}^{*}\right\|_{H, \varepsilon} \\
& =\left\|\nabla \varphi_{n, 0}\right\|_{H}^{2}+\left\|\widehat{\beta}_{\varepsilon}\left(\varphi_{n, 0}\right)\right\|_{L^{1}(\Omega)}+\rho\left\|\varphi_{n, 0}-\varphi_{n}^{*}\right\|_{H, \varepsilon} \\
& \quad+\int_{0}^{t}\left(-\pi\left(\varphi_{n}\right)+\gamma \partial_{t} w_{n}, \partial_{t} \varphi_{n}\right) \\
& \leq C\left(1+\rho+\varepsilon^{-1}\right)+\int_{0}^{t}\left(-\pi\left(\varphi_{n}\right)+\gamma \partial_{t} w_{n}, \partial_{t} \varphi_{n}\right) .
\end{aligned}
$$

The last integral can be estimated using Young inequality, the Lipschitz-continuity of $\pi$, and the first a priori estimate:

$$
\begin{aligned}
& \int_{0}^{t}\left(-\pi\left(\varphi_{n}\right)+\gamma \partial_{t} w_{n}, \partial_{t} \varphi_{n}\right) \\
& \leq \frac{1}{2}\left\|-\pi\left(\varphi_{n}\right)+\gamma \partial_{t} w_{n}\right\|_{L^{2}(0, T ; H)}^{2}+\frac{1}{2} \int_{0}^{t}\left\|\partial_{t} \varphi_{n}\right\|_{H}^{2} \\
& \leq C\left(1+\varepsilon^{-1}\right)+\frac{1}{2} \int_{0}^{t}\left\|\partial_{t} \varphi_{n}\right\|_{H}^{2} .
\end{aligned}
$$

Hence, we deduce

$$
\begin{aligned}
& \left\|\varphi_{n}\right\|_{H^{1}(0, T ; H) \cap L^{\infty}(0, T ; V)}+\left\|\widehat{\beta}_{\varepsilon}\left(\varphi_{n}\right)\right\|_{L^{\infty}\left(0, T ; L^{1}\right)} \\
& +\rho \sup _{t \in(0, T)}\left\|\varphi_{n}(t)-\varphi_{n}^{*}\right\|_{H, \varepsilon} \leq C\left(1+\rho^{1 / 2}+\varepsilon^{-2}\right) .
\end{aligned}
$$

Third a priori estimate. We define $g_{1}:[0, T] \rightarrow H$ as

$$
g_{1}=-\partial_{t} \varphi_{n}-\pi\left(\varphi_{n}\right)+\gamma \partial_{t} w_{n} .
$$

Clearly, it holds that $\left\|g_{1}\right\|_{L^{2}(0, T ; H)} \leq C\left(1+\rho+\varepsilon^{-1}\right)$, and we can rewrite equation (3.41) as

$$
\left(-\triangle \varphi_{n}+\beta_{\varepsilon}\left(\varphi_{n}\right)+\rho \operatorname{Sign}_{\varepsilon}\left(\varphi_{n}-\varphi_{n}^{*}\right), v\right)=\left(g_{1}, v\right) .
$$

We take $v=-\triangle \varphi_{n}$ and obtain

$$
\begin{aligned}
& \left\|\Delta \varphi_{n}\right\|_{H}^{2}+\int_{\Omega} \beta_{\varepsilon}^{\prime}\left(\varphi_{n}\right)\left|\nabla \varphi_{n}\right|^{2}+\rho\left(\nabla \operatorname{Sign}_{\varepsilon}\left(\varphi_{n}-\varphi_{n}^{*}\right), \nabla\left(\varphi_{n}-\varphi_{n}^{*}\right)\right) \\
& =\left(g_{1}, \triangle \varphi_{n}\right)+\rho\left(\operatorname{Sign}_{\varepsilon}\left(\varphi_{n}-\varphi_{n}^{*}\right), \triangle \varphi_{n}^{*}\right) \\
& \leq \frac{1}{2}\left\|g_{1}\right\|_{H}^{2}+\frac{1}{2}\left\|\triangle \varphi_{n}\right\|_{H}^{2}+\rho\left\|\Delta \varphi_{n}^{*}\right\|_{H} .
\end{aligned}
$$


Since the integral in the left-hand side of the equation above is non negative, we can forget about it. We can also cancel the third term, because

$$
\left(\nabla \operatorname{Sign}_{\varepsilon}\left(\varphi_{n}-\varphi_{n}^{*}\right), \nabla\left(\varphi_{n}-\varphi_{n}^{*}\right)\right)=\frac{\left\|\nabla\left(\varphi_{n}-\varphi_{n}^{*}\right)\right\|_{H}^{2}}{\max \left\{\varepsilon,\left\|\varphi_{n}-\varphi_{n}^{*}\right\|_{H}\right\}} \geq 0
$$

We integrate over time, obtaining

$$
\left\|\triangle \varphi_{n}\right\|_{L^{2}(0, T ; H)} \leq C\left(1+\rho^{1 / 2}+\varepsilon^{-1}\right) .
$$

By comparison and the elliptic regularity, we deduce that

$$
\begin{aligned}
& \left\|\varphi_{n}\right\|_{L^{2}(0, T ; W)}+\left\|\beta_{\varepsilon}\left(\varphi_{n}\right)+\rho \operatorname{Sign}_{\varepsilon}\left(\varphi_{n}-\varphi_{n}^{*}\right)\right\|_{L^{2}(0, T ; H)} \\
& \leq C\left(1+\rho^{1 / 2}+\varepsilon^{-1}\right) .
\end{aligned}
$$

Fourth a priori estimate. We define $\eta_{n}:[0, T] \rightarrow V_{n}$ as

$$
\eta_{n}(t):=\partial_{t} w_{n}(t)+l \varphi_{n}(t), \quad t \in[0, T] .
$$

Thanks to the estimate (3.44) we have that

$$
\left\|\eta_{n}\right\|_{L^{\infty}(0, T ; H)} \leq C\left(1+\varepsilon^{-1}\right) .
$$

Moreover, equation 2.5 implies

$$
\left\|\eta_{n}(0)\right\|_{V}=\left\|\vartheta_{0, n}+l \varphi_{0, n}\right\|_{V} \leq C
$$

We rewrite equation (3.40) as

$$
\left(\partial_{t} \eta_{n}-\kappa \partial_{t} \triangle w_{n}-\tau \triangle w_{n}, v\right)=\left(f_{n}, v\right) .
$$

Thus, we test the equation above with $v=-\Delta \eta_{n}(t)$ and we integrate over time finding

$$
\begin{aligned}
& \frac{1}{2}\left\|\nabla \eta_{n}(t)\right\|_{H}^{2}+\kappa \int_{0}^{t}\left\|\partial_{t} \triangle w_{n}\right\|_{H}^{2}+\frac{\tau}{2}\left\|\Delta w_{n}\right\|_{H}^{2} \\
& \leq C+\kappa l \int_{0}^{t}\left(\partial_{t} \triangle w_{n}, \triangle \varphi_{n}\right)+\tau l \int_{0}^{t}\left(\triangle w_{n}, \triangle \varphi_{n}\right)-\int_{0}^{t}\left(f_{n}, \triangle \eta_{n}\right) .
\end{aligned}
$$

The terms in the right-hand side of the inequality above can be controlled easily

$$
\begin{aligned}
& \kappa l \int_{0}^{t}\left(\partial_{t} \triangle w_{n}, \triangle \varphi_{n}\right) \leq \int_{0}^{t} \frac{\kappa}{2}\left\|\partial_{t} \triangle w_{n}\right\|_{H}^{2}+\int_{0}^{t} \frac{l^{2}}{2 \kappa}\left\|\Delta \varphi_{n}\right\|_{H}^{2}, \\
& \int_{0}^{t}\left(\triangle w_{n}, \triangle \varphi_{n}\right) \leq \frac{1}{2} \int_{0}^{t}\left(\left\|\triangle w_{n}\right\|_{H}^{2}+\left\|\Delta \varphi_{n}\right\|_{H}^{2}\right), \\
& \int_{0}^{t}\left(f_{n}, \triangle \eta_{n}\right) \leq C+\frac{\kappa}{4} \int_{0}^{t}\left\|\partial_{t} \triangle w_{n}\right\|_{H}^{2} .
\end{aligned}
$$

Hence, we infer

$$
\left\|\nabla \eta_{n}\right\|_{L^{\infty}(0, T ; H)}+\left\|\partial_{t} \triangle w_{n}\right\|_{L^{2}(0, T ; H)} \leq C\left(1+\rho^{1 / 2}+\varepsilon^{-1}\right),
$$

which, together with elliptic regularity and the estimate (3.45), implies

$$
\left\|\partial_{t} w_{n}\right\|_{L^{\infty}(0, T ; V)}+\left\|\partial_{t} w_{n}\right\|_{L^{2}(0, T ; W)} \leq C\left(1+\rho^{1 / 2}+\varepsilon^{-1}\right) .
$$

Finally, as $w_{0, n}$ is bounded in $W$, we conclude that

$$
\left\|w_{n}\right\|_{W^{1, \infty}(0, T ; V) \cap H^{1}(0, T ; W)} \leq C\left(1+\rho^{1 / 2}+\varepsilon^{-1}\right) .
$$

By comparison in (3.40), it follows that

$$
\left\|w_{n}\right\|_{H^{2}(0, T ; H) \cap W^{1, \infty}(0, T ; V) \cap H^{1}(0, T ; W)} \leq C\left(1+\rho^{1 / 2}+\varepsilon^{-1}\right) .
$$


Passage to the limit. The arguments used in the previous proof for the passage to the limit in the Faedo-Galerkin scheme and as $\varepsilon \rightarrow 0$ are still perfectly working. First, passing to the limit with respect to $n$ in (3.40)

$$
\begin{aligned}
& \left(\partial_{t}^{2} w_{\varepsilon}+l \partial_{t} \varphi_{\varepsilon}-\kappa \Delta \partial_{t} w_{\varepsilon}-\tau \Delta w_{\varepsilon}, v\right)=(f, v), \\
& \forall v \in H, \text { a.e. in }[0, T], \\
& \left(\partial_{t} \varphi_{\varepsilon}-\triangle \varphi_{\varepsilon}+\xi_{\varepsilon}+\pi\left(\varphi_{\varepsilon}\right)+\rho \sigma_{\varepsilon}, v\right)=\gamma\left(\partial_{t} w_{\varepsilon}, v\right), \\
& \forall v \in H, \text { a.e. in }[0, T], \\
& \partial_{t} w_{\varepsilon}(0)=\vartheta_{0}, \quad w_{\varepsilon}(0)=w_{0}, \quad \varphi_{\varepsilon}(0)=\varphi_{0},
\end{aligned}
$$

where

$$
\xi_{\varepsilon}:=\beta_{\varepsilon}\left(\varphi_{\varepsilon}\right) \quad \text { and } \quad \sigma_{\varepsilon}:=\operatorname{Sign}_{\varepsilon}\left(\varphi_{\varepsilon}-\varphi^{*}\right) .
$$

We want to replicate the a priori estimate for this new setting. As we have already pointed out, the only dependence on $\varepsilon$ are given via the estimate (3.43). We observe that (cf. (3.4))

$$
\left\|\beta_{\varepsilon}\left(\varphi^{*}\right)\right\|_{H} \leq\left\|\beta^{\circ}\left(\varphi^{*}\right)\right\|_{H}
$$

thus estimate (3.44) improves to

$$
\begin{aligned}
& \left\|w_{\varepsilon}\right\|_{W^{1, \infty}(0, T ; H) \cap H^{1}(0, T ; V)}+\left\|\varphi_{\varepsilon}\right\|_{L^{\infty}(0, T ; H) \cap L^{2}(0, T ; V)} \\
& +\rho \int_{0}^{T}\left\|\varphi_{\varepsilon}-\varphi^{*}\right\|_{H, \varepsilon} \leq C,
\end{aligned}
$$

and we have just made the first a priori estimate independent of $\varepsilon$. Having removed the dependence on $\varepsilon$ in the first estimate, all other estimates can be replicated obtaining

$$
\begin{aligned}
& \left\|\varphi_{\varepsilon}\right\|_{H^{1}(0, T ; H) \cap L^{\infty}(0, T ; V)}+\left\|\widehat{\beta}_{\varepsilon}\left(\varphi_{\varepsilon}\right)\right\|_{L^{\infty}\left(0, T ; L^{1}\right)} \\
& +\rho \sup _{t \in(0, T)}\left\|\varphi_{\varepsilon}(t)-\varphi^{*}\right\|_{H, \varepsilon} \leq C\left(1+\rho^{1 / 2}\right), \\
& \left\|\varphi_{\varepsilon}\right\|_{L^{2}(0, T ; W)}+\left\|\xi_{\varepsilon}+\rho \sigma_{\varepsilon}\right\|_{L^{2}(0, T ; H)} \leq C\left(1+\rho^{1 / 2}\right), \\
& \left\|w_{\varepsilon}\right\|_{H^{2}(0, T ; H) \cap W^{1, \infty}(0, T ; V) \cap H^{1}(0, T ; W)} \leq C\left(1+\rho^{1 / 2}\right) .
\end{aligned}
$$

Moreover, because of the definition of the Sign operator, $\sigma_{\varepsilon}(t)$ is bounded, uniformly with respect to $t$ and $\varepsilon$, i.e.,

$$
\left\|\sigma_{\varepsilon}\right\|_{L^{\infty}(0, T ; H)} \leq 1
$$

By comparison we have that $\left\|\xi_{\varepsilon}\right\|_{L^{2}(0, T ; H)} \leq C(1+\rho)$. Like for Problem (A), we can use standard compactness results and extract a subsequence such that

$$
\left(w_{\varepsilon}, \varphi_{\varepsilon}, \xi_{\varepsilon}, \sigma_{\varepsilon}\right) \rightarrow(w, \varphi, \xi, \sigma)
$$

in a suitable topology for the spaces (2.6) (2.9) where the solutions are set. Moreover, we have that

$$
\varphi_{\varepsilon} \rightarrow \varphi \quad \text { in } C^{0}([0, T] ; H) .
$$

We conclude that Problem (B) admits a solution and that (2.34)-(2.35) hold, using the same argument used in Problem (A).

The uniqueness of the solution follows immediately from Theorem 2.8, and it is treated in Section 5.

\section{Further regularity}

This section contains the proofs of Theorems 2.2 and 2.9 . For the sake of clarity, both proofs can be divided in two parts. In the former part we use the notations of the Faedo-Galerkin scheme to prove that the limit function $\varphi_{\varepsilon}$ is more regular (in particular $\varphi_{\varepsilon} \in H^{1}(0, T ; V)$ ). In the latter part, the desired estimates (2.27) and (2.39) are shown for the approximate solution $\varphi_{\varepsilon}$. Using the usual compactness argument and the lower semi-continuity of the norm, the estimates (2.27) and (2.39) will follow automatically. In the present proofs, $C$ still denotes a positive constant independent of $\rho$ and $\varepsilon$. 


\subsection{Further regularity for Problem (A)}

We now prove Theorem 2.2 We consider equation (3.12) and note that: 1) the functions $\triangle \varphi_{n}$ and $\partial_{t} w_{n}$ are derivable and their derivatives are $\triangle \partial_{t} \varphi_{n}$ and $\partial_{t}^{2} w_{n}$, respectively; 2) for all $v \in V_{n}$, the functions

$$
t \mapsto\left(\beta_{\varepsilon}\left(\varphi_{n}(t)\right), v\right) \quad \text { and } \quad t \mapsto\left(\pi\left(\varphi_{n}(t)\right), v\right)
$$

are Lipschitz-continuous, thus derivable a.e. in $(0, T)$ with derivative

$$
\left(\beta_{\varepsilon}^{\prime}\left(\varphi_{n}(t)\right) \partial_{t} \varphi_{n}(t), v\right) \quad \text { and } \quad\left(\pi^{\prime}\left(\varphi_{n}(t)\right) \partial_{t} \varphi_{n}(t), v\right)
$$

respectively. Hence, $\partial_{t} \varphi_{n}$ is Lipschitz-continuous by comparison and we can derive (in weak sense) equation (3.12) obtaining

$$
\left(\partial_{t}^{2} \varphi_{n}-\triangle \partial_{t} \varphi_{n}+\beta_{\varepsilon}^{\prime}\left(\varphi_{n}\right) \partial_{t} \varphi_{n}, v\right)=\left(g_{4}, v\right), \quad \forall v \in V_{n}, \text { a.e. in }(0, T),
$$

where

$$
g_{4}:=-\pi^{\prime}\left(\varphi_{\varepsilon}\right) \partial_{t} \varphi_{n}+\gamma \partial_{t}^{2} w_{n}
$$

Clearly $\left\|g_{4}\right\|_{L^{2}(0, T ; H)} \leq C\left(1+\varepsilon^{-1}+\rho^{1 / 2}\right)$, as $\pi^{\prime}$ is bounded. We take $v=\partial_{t} \varphi_{\varepsilon}$ in equation (4.1) and we integrate over $(0, t)$ obtaining

$$
\begin{aligned}
& \frac{1}{2}\left\|\partial_{t} \varphi_{n}(t)\right\|_{H}^{2}+\int_{0}^{t}\left\|\nabla \partial_{t} \varphi_{n}\right\|_{H}^{2}+\int_{Q_{t}} \beta_{\varepsilon}^{\prime}\left(\varphi_{n}\right)\left|\partial_{t} \varphi_{n}\right|^{2} \\
& =\frac{1}{2}\left\|\partial_{t} \varphi_{n}(0)\right\|_{H}^{2}+\int_{0}^{t}\left(g_{4}, \partial_{t} \varphi_{n}\right) \\
& \leq \frac{1}{2}\left\|\partial_{t} \varphi_{n}(0)\right\|_{H}^{2}+\frac{1}{2} \int_{0}^{t}\left\|g_{4}\right\|_{H}^{2}+\frac{1}{2} \int_{0}^{t}\left\|\partial_{t} \varphi_{n}\right\|_{H}^{2} \\
& \leq \frac{1}{2}\left\|\partial_{t} \varphi_{n}(0)\right\|_{H}^{2}+\frac{1}{2} \int_{0}^{t}\left\|\partial_{t} \varphi_{n}\right\|_{H}^{2}+C\left(1+\varepsilon^{-2}+\rho\right) .
\end{aligned}
$$

Since $\beta_{\varepsilon}$ is monotone, we have that $\beta_{\varepsilon}^{\prime} \geq 0$ implies $\int_{Q_{t}} \beta_{\varepsilon}^{\prime}\left(\varphi_{n}\right)\left|\partial_{t} \varphi_{n}\right|^{2} \geq 0$. At this point, we want to use the Gronwall lemma to control $\left\|\partial_{t} \varphi_{n}\right\|_{L^{2}(0, T ; H)}$. The most delicate part is to find a bound on $\left\|\partial_{t} \varphi_{n}(0)\right\|_{H}$. Using again equation (3.12) we compute

$$
\begin{aligned}
\left\|\partial_{t} \varphi_{n}(0)\right\|_{H} & =\left\|\gamma \vartheta_{0, n}+\triangle \varphi_{0, n}-P_{n}\left(\beta_{\varepsilon}\left(\varphi_{0, n}\right)\right)-P_{n}\left(\pi\left(\varphi_{0, n}\right)\right)\right\|_{H} \\
& \leq \gamma\left\|\vartheta_{0, n}\right\|_{H}+\left\|\triangle \varphi_{0, n}\right\|_{H}+\left\|P_{n}\left(\pi\left(\varphi_{0, n}\right)\right)\right\|_{H}+\left\|P_{n}\left(\beta_{\varepsilon}\left(\varphi_{0, n}\right)\right)\right\|_{H} \\
& \leq \gamma\left\|\vartheta_{0}\right\|_{H}+\left\|\triangle \varphi_{0}\right\|_{H}+C\left\|\varphi_{0}\right\|_{H}+\varepsilon^{-1}\left\|\varphi_{0}\right\|_{H} \leq C\left(1+\varepsilon^{-1}\right),
\end{aligned}
$$

where the fact that $\beta_{\varepsilon}$ is $\varepsilon^{-1}$-Lipschitz-continous and the hypothesis $\varphi_{0} \in W$ have been taken into account. We incidentally note that we have not yet used the hypothesis $\beta^{\circ}\left(\varphi_{0}\right) \in H$. Owing to the Gronwall lemma, we obtain

$$
\left\|\varphi_{n}\right\|_{W^{1, \infty}(0, T ; H) \cap H^{1}(0, T ; V)} \leq C\left(1+\varepsilon^{-1}+\rho^{1 / 2}\right) .
$$

In view of equations (3.22) and (3.24), we additionally have that

$$
\varphi_{n} \rightarrow \varphi_{\varepsilon} \quad \text { weakly* in } W^{1, \infty}(0, T ; H) \cap H^{1}(0, T ; V) .
$$

This proves that $\varphi_{\varepsilon}$ belongs to $W^{1, \infty}(0, T ; H) \cap H^{1}(0, T ; V)$.

In this second part we refine our argument, removing the dependence on $\varepsilon$ in the estimate (4.4). Like the former part of this proof, we want to derive equation (3.28). Since nothing ensures the weakderivability of $\triangle \varphi_{\varepsilon}$, we take $v \in V$ and rewrite (3.28) using the Gauss theorem

$$
\left(\partial_{t} \varphi_{\varepsilon}, v\right)+\left(\nabla \varphi_{\varepsilon}, \nabla v\right)+\left(\beta_{\varepsilon}\left(\varphi_{\varepsilon}\right), v\right)=\left(-\pi\left(\varphi_{\varepsilon}\right)+\gamma \partial_{t} w_{\varepsilon}, v\right)
$$

At this point, since $\varphi_{\varepsilon} \in H^{1}(0, T ; V)$ and the considerations on the weak-derivability of $\beta_{\varepsilon}\left(\varphi_{\varepsilon}\right), \pi\left(\varphi_{\varepsilon}\right)$ and $\partial_{t} w_{\varepsilon}$ remain valid, $\partial_{t} \varphi_{\varepsilon}$ is derivable with respect to time. We derive the above equation finding

$$
\left(\partial_{t}^{2} \varphi_{\varepsilon}, v\right)+\left(\nabla \partial_{t} \varphi_{\varepsilon}, \nabla v\right)+\left(\beta_{\varepsilon}^{\prime}\left(\varphi_{\varepsilon}\right) \partial_{t} \varphi_{\varepsilon}, v\right)=\left(\tilde{g}_{4}, v\right), \quad \forall v \in V \text {, a.e. in }(0, T),
$$


where $\tilde{g}_{4}$ is defined in the same way as $g_{4}$ and it satisfies $\left\|\tilde{g}_{4}\right\|_{L^{2}(0, T ; H)} \leq C\left(1+\rho^{1 / 2}\right)$. We take $v=\partial_{t} \varphi_{\varepsilon}$ and after some calculations carried out as in the former part we arrive at

$$
\frac{1}{2}\left\|\partial_{t} \varphi_{\varepsilon}(t)\right\|_{H}^{2}+\int_{0}^{t}\left\|\nabla \partial_{t} \varphi_{\varepsilon}\right\|_{H}^{2} \leq \frac{1}{2}\left\|\partial_{t} \varphi_{\varepsilon}(0)\right\|_{H}^{2}+C(1+\rho)+\int_{0}^{t}\left\|\partial_{t} \varphi_{\varepsilon}\right\|_{H}^{2} .
$$

Now, using the hypothesis $\beta^{\circ}\left(\varphi_{0}\right) \in H$ we deduce that

$$
\left\|\beta_{\varepsilon}\left(\varphi_{0}\right)\right\|_{H} \leq\left\|\beta^{\circ}\left(\varphi_{0}\right)\right\|_{H} \leq C .
$$

Hence, arguing as in equation (4.3), we have that $\left\|\partial_{t} \varphi_{\varepsilon}(0)\right\|_{H} \leq C$ and use the Gronwall lemma to deduce that

$$
\left\|\varphi_{\varepsilon}\right\|_{W^{1, \infty}(0, T ; H) \cap H^{1}(0, T ; V)} \leq C\left(1+\rho^{1 / 2}\right) .
$$

By comparison, we find $\left\|-\triangle \varphi_{\varepsilon}+\xi_{\varepsilon}\right\|_{L^{\infty}(0, T ; H)} \leq C\left(1+\rho^{1 / 2}\right)$, whence, by the same argument as in the second a priori estimate (3.17), we conclude that $\Delta \varphi_{\varepsilon}, \xi_{\varepsilon} \in L^{\infty}(0, T ; H)$ and that

$$
\left\|\triangle \varphi_{\varepsilon}\right\|_{L^{\infty}(0, T ; H)}+\left\|\xi_{\varepsilon}\right\|_{L^{\infty}(0, T ; H)} \leq C\left(1+\rho^{1 / 2}\right) .
$$

\subsection{Further regularity for Problem (B)}

Since the proof of Theorem 2.9 is based on the same idea of the previous proof, the present proof is just sketched and we will focus only on the differences. We consider equation (3.41) and, like before, we want to derive it with respect to the time. The terms $\Delta \varphi_{n}, \beta_{\varepsilon}\left(\varphi_{n}\right), \pi\left(\varphi_{n}\right)$, and $\partial_{t} w$ are weaklydifferentiable by the considerations made in the previous subsection. If the term $\rho \operatorname{Sign}_{\varepsilon}\left(\varphi_{n}-\varphi_{n}^{*}\right)$ were weakly-differentiable, we could carry out the proof as before; the weak-derivability of this term is shown in the following lemma.

Lemma 4.1. Let $\varepsilon>0$ and $u \in H^{1}(0, T ; H)$. Let $A=\left\{t \in[0, T]:\|\varphi(t)\|_{H} \leq \varepsilon\right\}$ and $B=[0, T] \backslash A$. Then $\operatorname{Sign}_{\varepsilon}(u) \in H^{1}(0, T ; H)$ and

$$
\frac{\mathrm{d}}{\mathrm{d} t} \operatorname{Sign}_{\varepsilon}(u)= \begin{cases}\frac{u_{t}}{\varepsilon} & \text { a.e. in } A, \\ \frac{u_{t}}{\|u\|_{H}}-\frac{\left(u, u_{t}\right)}{\|u\|_{H}^{3}} u & \text { a.e. in } B .\end{cases}
$$

Moreover, we have that

$$
\left(\frac{\mathrm{d}}{\mathrm{d} t} \operatorname{Sign}_{\varepsilon}(u), u_{t}\right) \geq 0 \quad \text { a.e. in }[0, T] .
$$

Proof. Let $l(t)=1 / \max \left\{\varepsilon,\|u(t)\|_{H}\right\}$. By the Stampacchia theorem, this function belongs to $H^{1}(0, T)$ and its derivative is

$$
\frac{\mathrm{d} l}{\mathrm{~d} t}= \begin{cases}0 & \text { a.e. in } A, \\ -\frac{\left(u, u_{t}\right)}{\|u\|_{H}^{3}} & \text { a.e. in } B .\end{cases}
$$

By equation (3.6), we have that $\operatorname{Sign}_{\varepsilon}(u(t))=l(t) u(t)$. Hence, $\operatorname{Sign}_{\varepsilon}(u)$ is differentiable by Leibniz rule and its derivative is

$$
\frac{\mathrm{d}}{\mathrm{d} t} \operatorname{Sign}_{\varepsilon}(u)=l_{t} u+l u_{t}= \begin{cases}\frac{u_{t}}{\varepsilon} & \text { a.e. in } A, \\ -\frac{\left(u_{t}, u\right)}{\|u\|_{H}^{3}} u+\frac{u_{t}}{\|u\|_{H}} & \text { a.e. in } B .\end{cases}
$$

Inequality (4.9) is trivial for a.a. $t \in A$; on $B$ we use the Cauchy-Schwartz inequality obtaining

$$
\left(\frac{\mathrm{d}}{\mathrm{d} t} \operatorname{Sign}_{\varepsilon}(u), u_{t}\right)=-\frac{\left(u_{t}, u\right)^{2}}{\|u\|_{H}^{3}}+\frac{\left\|u_{t}\right\|_{H}^{2}}{\|u\|_{H}} \geq-\frac{\left\|u_{t}\right\|_{H}^{2}\|u\|_{H}^{2}}{\|u\|_{H}^{3}}+\frac{\left\|u_{t}\right\|_{H}^{2}}{\|u\|_{H}}=0 .
$$


At this point, taking Lemma 4.1 into account, we have that $\varphi_{t}$ is weakly-differentiable by comparison and we can derive equation (3.41) obtaining

$$
\begin{aligned}
& \left(\partial_{t}^{2} \varphi_{n}-\Delta \partial_{t} \varphi_{n}+\beta_{\varepsilon}^{\prime}\left(\varphi_{n}\right) \partial_{t} \varphi_{n}+\rho \frac{\mathrm{d}}{\mathrm{d} t} \operatorname{Sign}_{\varepsilon}\left(\varphi_{n}-\varphi_{n}^{*}\right), v\right)=\left(g_{4}, v\right) \\
& \forall v \in V_{n}, \text { a.e. in }(0, T),
\end{aligned}
$$

where

$$
g_{4}:=-\pi^{\prime}\left(\varphi_{\varepsilon}\right) \partial_{t} \varphi_{n}+\gamma \partial_{t}^{2} w_{n}, \quad \text { with }\left\|g_{4}\right\|_{L^{2}(0, T ; H)} \leq C\left(1+\varepsilon^{-1}+\rho^{1 / 2}\right) .
$$

We take $v=\partial_{t} \varphi_{\varepsilon}$ in equation (4.12) and integrate over $(0, t)$ obtaining

$$
\begin{aligned}
& \frac{1}{2}\left\|\partial_{t} \varphi_{n}(t)\right\|_{H}^{2}+\int_{0}^{t}\left\|\nabla \partial_{t} \varphi_{n}\right\|_{H}^{2}+\int_{Q_{t}} \beta_{\varepsilon}^{\prime}\left(\varphi_{n}\right)\left|\partial_{t} \varphi_{n}\right|^{2} \\
& +\rho \int_{0}^{t}\left(\left(\operatorname{Sign}_{\varepsilon}\left(\varphi_{n}-\varphi_{n}^{*}\right)\right)_{t}, \partial_{t} \varphi_{n}\right) \\
& =\frac{1}{2}\left\|\partial_{t} \varphi_{n}(0)\right\|_{H}^{2}+\int_{0}^{t}\left(g_{4}, \partial_{t} \varphi_{n}\right) \\
& \leq \frac{1}{2}\left\|\partial_{t} \varphi_{n}(0)\right\|_{H}^{2}+\frac{1}{2} \int_{0}^{t}\left\|\partial_{t} \varphi_{n}\right\|_{H}^{2}+C\left(1+\varepsilon^{-2}+\rho\right) .
\end{aligned}
$$

The inequality above differs from (4.2) only for the presence of the term involving the derivative of $\operatorname{Sign}_{\varepsilon}$. This term is non-negative due to inequality (4.9). We have to control the term $\left\|\partial_{t} \varphi_{n}(0)\right\|_{H}$, which can be treated similarly as (4.3), with the only difference that now we have the additional contribution

$$
\rho\left\|\operatorname{Sign}_{\varepsilon}\left(\varphi_{n}-\varphi_{n}^{*}\right)\right\|_{H} \leq \rho
$$

on the right-hand side. Then, using (4.13) and applying the Gronwall lemma, we easily arrive at

$$
\left\|\varphi_{n}\right\|_{W^{1, \infty}(0, T ; H) \cap H^{1}(0, T ; V)} \leq C\left(1+\varepsilon^{-1}+\rho\right) .
$$

Possibly taking subsequences, like in (4.5) we have that

$$
\varphi_{n} \rightarrow \varphi_{\varepsilon} \quad \text { weakly* in } W^{1, \infty}(0, T ; H) \cap H^{1}(0, T ; V),
$$

thus $\varphi_{\varepsilon} \in W^{1, \infty}(0, T ; H) \cap H^{1}(0, T ; V)$.

We rewrite equation (3.50) as

$$
\begin{aligned}
& \left(\partial_{t} \varphi_{\varepsilon}, v\right)+\left(\nabla \varphi_{\varepsilon}, \nabla v\right)+\left(\beta_{\varepsilon}\left(\varphi_{\varepsilon}\right), v\right)+\rho\left(\operatorname{Sign}_{\varepsilon}\left(\varphi_{\varepsilon}-\varphi^{*}\right), v\right) \\
& =\left(-\pi\left(\varphi_{\varepsilon}\right)+\gamma \partial_{t} w_{\varepsilon}, v\right)
\end{aligned}
$$

derive it, and test with $v=\partial_{t} \varphi_{\varepsilon}$. After few calculations in the same style as before, the estimate (4.14) improves to

$$
\left\|\varphi_{\varepsilon}\right\|_{W^{1, \infty}(0, T ; H) \cap H^{1}(0, T ; V)} \leq C(1+\rho) .
$$

By comparison, we find $\left\|-\triangle \varphi_{\varepsilon}+\xi_{\varepsilon}\right\|_{L^{\infty}(0, T ; H)} \leq C(1+\rho)$. We multiply $-\triangle \varphi_{\varepsilon}+\xi_{\varepsilon}$ by $-\triangle \varphi_{\varepsilon}$, obtaining

$$
\begin{aligned}
& \left\|\triangle \varphi_{\varepsilon}\right\|_{H}^{2}+\int_{\Omega} \beta_{\varepsilon}^{\prime}\left|\nabla \varphi_{\varepsilon}\right|^{2} \leq\left\|\Delta \varphi_{\varepsilon}\right\|_{H}\left\|-\Delta \varphi_{\varepsilon}+\xi_{\varepsilon}\right\|_{H} \\
& \leq \frac{1}{2}\left\|\triangle \varphi_{\varepsilon}\right\|_{H}^{2}+C\left(1+\rho^{2}\right) \quad \text { a.e. in }(0, T) .
\end{aligned}
$$

Thus, we conclude that $\left\|\triangle \varphi_{\varepsilon}\right\|_{L^{\infty}(0, T ; W)} \leq C(1+\rho)$ by elliptic regularity and $\left\|\xi_{\varepsilon}\right\|_{L^{\infty}(0, T ; H)} \leq C(1+\rho)$ by comparison.

\section{Continuous dependence of the solutions}

We prove now Theorems 2.3 and 2.8 . 
Continuous dependence of the solutions for Problem (A). In order to simplify the notation, we let $\vartheta_{0}=\vartheta_{0,1}-\vartheta_{0,2}$ and analogously we define $w_{0}, \varphi_{0}, f, w, \varphi, \xi$ and $\sigma$. In this proof, $C$ denotes a time-to-time-different, positive, large-enough constant independent of the just-said data and of $\rho$.

It is clear that

$$
\begin{aligned}
& \left(w_{t}+l \varphi\right)_{t}-\kappa \Delta w_{t}-\tau \Delta w+\rho \sigma=f, \\
& \varphi_{t}-\triangle \varphi+\xi+\pi\left(\varphi_{1}\right)-\pi\left(\varphi_{2}\right)=\gamma w_{t} .
\end{aligned}
$$

We multiply equations (5.1) and (5.2) by $\left(w_{t}+l \varphi\right)$ and $\kappa l^{2} \varphi$ respectively, sum up and integrate over $\Omega$ obtaining

$$
\begin{aligned}
& \frac{1}{2} \frac{\mathrm{d}}{\mathrm{d} t}\left\|w_{t}+l \varphi\right\|_{H}^{2}+\kappa\left\|\nabla w_{t}\right\|_{H}^{2}+\kappa l\left(\nabla w_{t}, \nabla \varphi\right)+\frac{\tau}{2} \frac{\mathrm{d}}{\mathrm{d} t}\|\nabla w\|_{H}^{2} \\
& +\tau l(\nabla w, \nabla \varphi)+\rho\left(\sigma, w_{t}+l \varphi\right)_{H}+\frac{\kappa l^{2}}{2} \frac{\mathrm{d}}{\mathrm{d} t}\|\varphi\|_{H}^{2} \\
& +\kappa l^{2}\|\nabla \varphi\|_{H}^{2}+\kappa l^{2}(\xi, \varphi)_{H}+\kappa l^{2}\left(\pi\left(\varphi_{1}\right)-\pi\left(\varphi_{2}\right), \varphi\right)_{H} \\
& =\left(f, w_{t}\right)+l(f, \varphi)+\gamma \kappa l^{2}\left(w_{t}, \varphi\right) .
\end{aligned}
$$

We rearrange and use the Lipschitz-continuity of $\pi$, equations (2.11) and (2.13), and the monotonicity of Sign and $\beta$ to infer that

$$
\begin{aligned}
& \frac{1}{2} \frac{\mathrm{d}}{\mathrm{d} t}\left\|w_{t}+l \varphi\right\|_{H}^{2}+\frac{\tau}{2} \frac{\mathrm{d}}{\mathrm{d} t}\|\nabla w\|_{H}^{2}+\frac{\kappa l^{2}}{2} \frac{\mathrm{d}}{\mathrm{d} t}\|\varphi\|_{H}^{2} \\
& +\kappa\left(\left\|\nabla w_{t}\right\|_{H}^{2}+l\left(\nabla w_{t}, \nabla \varphi\right)+l^{2}\|\nabla \varphi\|_{H}^{2}\right) \\
& \leq\left(f, w_{t}\right)+l(f, \varphi)+\gamma \kappa l^{2}\left(w_{t}, \varphi\right)+C\|\varphi\|_{H}^{2}-\tau l(\nabla w, \nabla \varphi) .
\end{aligned}
$$

At this point, the Young inequality and the fact that

$$
l\left(\nabla w_{t}, \nabla \varphi\right) \geq-\frac{1}{2}\left(\left\|\nabla w_{t}\right\|_{H}^{2}+l^{2}\|\nabla \varphi\|_{H}^{2}\right)
$$

allow us to deduce

$$
\begin{aligned}
& \frac{1}{2} \frac{\mathrm{d}}{\mathrm{d} t}\left\|w_{t}+l \varphi\right\|_{H}^{2}+\frac{\kappa l^{2}}{2} \frac{\mathrm{d}}{\mathrm{d} t}\|\varphi\|_{H}^{2}+\frac{\tau}{2} \frac{\mathrm{d}}{\mathrm{d} t}\|\nabla w\|_{H}^{2} \\
& +\frac{\kappa}{2}\left(\left\|\nabla w_{t}\right\|_{H}^{2}+l^{2}\|\nabla \varphi\|_{H}^{2}\right) \\
& \leq C\|f(t)\|_{H}^{2}+C\left(\left\|w_{t}\right\|_{H}^{2}+\|\varphi\|_{H}^{2}\right)+\frac{\kappa l^{2}}{4}\|\nabla \varphi\|_{H}^{2}+\frac{\tau^{2}}{\kappa}\|\nabla w\|_{H}^{2} .
\end{aligned}
$$

We integrate between 0 and $t$

$$
\begin{aligned}
& \frac{1}{2}\left\|w_{t}(t)+l \varphi(t)\right\|_{H}^{2}+\frac{\kappa l^{2}}{2}\|\varphi(t)\|_{H}^{2}+\frac{\tau}{2}\|\nabla w(t)\|_{H}^{2} \\
& +\frac{\kappa}{2} \int_{0}^{t}\left\|\nabla w_{t}\right\|_{H}^{2}+\frac{\kappa l^{2}}{4} \int_{0}^{t}\|\nabla \varphi\|_{H}^{2} \\
& \leq C\|f\|_{L^{2}(0, T ; H)}^{2}+\frac{1}{2}\left\|\vartheta_{0}+l \varphi_{0}\right\|_{H}^{2}+\frac{\kappa l^{2}}{2}\left\|\varphi_{0}\right\|_{H}^{2}+\frac{\tau}{2}\left\|\nabla w_{0}\right\|_{H}^{2} \\
& \quad+C \int_{0}^{t}\left(\left\|w_{t}\right\|_{H}^{2}+\|\varphi\|_{H}^{2}\right)+\frac{\tau^{2}}{\kappa} \int_{0}^{t}\|\nabla w\|_{H}^{2} .
\end{aligned}
$$

Finally, we note that

$$
\begin{aligned}
& \frac{\kappa}{\kappa+2}\left\|w_{t}(t)\right\|_{H}^{2}+\frac{\kappa l^{2}}{2}\|\varphi(t)\|_{H}^{2} \leq\left\|w_{t}(t)+l \varphi(t)\right\|_{H}^{2}+\kappa l^{2}\|\varphi(t)\|_{H}^{2}, \\
&\left\|\vartheta_{0}+l \varphi_{0}\right\|_{H}^{2}+\kappa l^{2}\left\|\varphi_{0}\right\|_{H}^{2} \leq 2\left\|\vartheta_{0}\right\|_{H}^{2}+(\kappa+1) l^{2}\left\|\varphi_{0}\right\|_{H}^{2},
\end{aligned}
$$

and so we can apply the Gronwall Lemma finding

$$
\begin{aligned}
& \left\|w_{t}\right\|_{L^{\infty}(0, T ; H)}+\|\nabla w\|_{L^{\infty}(0, T ; H)}+\left\|\nabla w_{t}\right\|_{L^{2}(0, T ; H)} \\
& +\|\varphi\|_{L^{\infty}(0, T ; H)}+\|\nabla \varphi\|_{L^{2}(0, T ; H)} \\
& \leq C\left(\|f\|_{L^{2}(0, T ; H)}+\left\|\vartheta_{0}\right\|_{H}+\left\|\varphi_{0}\right\|_{H}+\left\|\nabla w_{0}\right\|_{H}\right) .
\end{aligned}
$$


As $w_{0}, \varphi_{0} \in V$, this implies that

$$
\begin{gathered}
\|w\|_{W^{1, \infty}(0, T ; H) \cap H^{1}(0, T ; V)}+\|\varphi\|_{L^{\infty}(0, T ; H) \cap L^{2}(0, T ; V)} \\
\leq C\left(\|f\|_{L^{2}(0, T ; H)}+\left\|\vartheta_{0}\right\|_{H}+\left\|w_{0}\right\|_{V}+\left\|\varphi_{0}\right\|_{H}\right),
\end{gathered}
$$

and the proof is complete.

We conclude this paragraph with the proof of Corollary 2.4. Assume that $\left(w_{i}, \varphi_{i}, \xi_{i}, \sigma_{i}\right), i=1,2$, are two solutions given by the existence Theorem 2.1. Since $l=\alpha$ we can apply the just-proven Theorem 2.3 with $\left(\vartheta_{0, i}, w_{0, i}, \varphi_{0, i}, f_{i}\right)=\left(\vartheta_{0}, w_{0}, \varphi_{0}, f\right)$. Hence, by the equation above we deduce that $w_{1}=w_{2}$ and $\varphi_{1}=\varphi_{2}$. By comparison, we conclude that $\sigma_{1}=\sigma_{2}$ and $\xi_{1}=\xi_{2}$.

Continuous dependence of the solutions for Problem (B). We do not give the proof of Theorem 2.8 since it goes along the same line of the previous one and no further idea arises. As before, continuous dependence implies uniqueness of the components $w$ and $\varphi$. If in addition $\beta$ is single-valued, $\xi$ is uniquely determined, thus, by comparison in (2.17), the function $\sigma$ is uniquely determined, as well.

\section{$6 \quad$ Existence of sliding modes}

This section is devoted to prove Theorems 2.5 and 2.10 In this section, we deal with the approximated solutions $\left(w_{\varepsilon}, \varphi_{\varepsilon}, \xi_{\varepsilon}, \sigma_{\varepsilon}\right)$ and we will take the limit as $\varepsilon \rightarrow 0$. Before going through the proofs of the Theorems, we show the following Lemma.

Lemma 6.1. Let $T, M>02 \psi_{0} \geq 0$, and let $\psi:[0, T] \rightarrow \mathbb{R}$ be an a non-negative, absolutely continuous function with $\psi(0)=\psi_{0}$. Let $A$ be the set

$$
A:=\{t \in[0, T]: \psi(t)>0\} .
$$

If $\psi^{\prime}(t) \leq-M$ a.e. in $A$, then the following conclusions hold true.

1) If $\psi_{0}=0$, then $\psi \equiv 0$.

2) If $M>\psi_{0} / T$, then there exist a time $T^{*} \in(0, T)$ such that

$$
T^{*} \leq \frac{\psi_{0}}{M}<T,
$$

as well as the function $\psi$ is strictly decreasing in $\left[0, T^{*}\right)$ and vanishes in $\left[T^{*}, T\right]$.

Proof. 1) Suppose on the contrary that $A$ is non empty. Let $B=(a, b)$ be a connected component of $A$. The function $\psi$ restricted to $B$ is strictly decreasing. Indeed, if $a<t_{0}<t_{1}<b$, we have that

$$
\psi\left(t_{1}\right)-\psi\left(t_{0}\right)=\int_{t_{0}}^{t_{1}} \psi^{\prime}(s) \mathrm{d} s \leq-M\left(t_{1}-t_{0}\right)<0 .
$$

We now take the limit as $t_{0} \rightarrow a$ obtaining

$$
\psi\left(t_{1}\right) \leq \lim _{t_{0} \rightarrow a} \psi\left(t_{0}\right)=\psi(a)=0,
$$

which is a contradiction for we assumed $\psi\left(t_{1}\right)>0$.

2) We may assume $\psi_{0}>0$, because the case $\psi_{0}=0$ follows directly from the former part with $T^{*}=0$. We define $T^{*}$ as

$$
T^{*}:=\sup \{t \in(0, T): \psi(s)>0 \text { for all } s \in(0, t)\} .
$$

By continuity of $\psi, T^{*}$ is well-defined and greater than 0 . Moreover, the interval $\left[0, T^{*}\right)$ is contained in $A$, hence we have that

thus

$$
\psi\left(T^{*}\right)-\psi(0)=\int_{0}^{T^{*}} \psi^{\prime}(t) \mathrm{d} t \leq-M T^{*},
$$

$$
T^{*} \leq \frac{\psi_{0}-\psi\left(T^{*}\right)}{M} \leq \frac{\psi_{0}}{M}<T .
$$

Note that $\psi$ is strictly decreasing in $\left[0, T^{*}\right)$ for what we have proven in 1$)$. It is clear that $\psi\left(T^{*}\right)=0$. Indeed, if on the contrary $\psi\left(T^{*}\right)>0$, then $\psi>0$ in $\left[0, T^{*}+\varepsilon\right)$ for a small $\varepsilon$ and the supremum in definition (6.3) fails. Finally, we define $\delta:\left[0, T-T^{*}\right] \rightarrow[0,+\infty)$ as $\delta(t)=\psi\left(t+T^{*}\right)$ and we use the first part of the lemma, deducing $\delta=0$, thus $\psi(t)=0$ for all $t \in\left[T^{*}, T\right]$.

\footnotetext{
${ }^{2}$ The first part of this lemma is still working if $M=0$.
} 


\subsection{Existence of sliding modes for Problem (A)}

We define for $\varepsilon>0, \eta_{\varepsilon}, g_{\varepsilon}:[0, T] \rightarrow H$ as

$$
\begin{aligned}
& \eta_{\varepsilon}:=\partial_{t} w_{\varepsilon}+\alpha \varphi_{\varepsilon}-\eta^{*}, \\
& g_{\varepsilon}:=\tau \triangle w_{\varepsilon}-\kappa \alpha \Delta \varphi_{\varepsilon}+(\alpha-l) \partial_{t} \varphi_{\varepsilon}-\kappa \Delta \eta^{*}+f .
\end{aligned}
$$

Analogously, we define $\eta=\partial_{t} w+\alpha \varphi-\eta^{*}$. Because of the estimates (3.29), (4.7), (4.6), and the hypothesis on the target function (2.15), we infer that $g_{\varepsilon} \in L^{\infty}(0, T ; H)$ and

$$
\left\|g_{\varepsilon}\right\|_{L^{\infty}(0, T ; H)} \leq C_{5}\left(1+\rho^{1 / 2}\right)
$$

where the constant $C_{5}$ is defined in equation (2.30). Recalling that $\sigma_{\varepsilon}=\operatorname{Sign}_{\varepsilon}\left(\eta_{\varepsilon}\right)$ we rewrite equation (3.27) as

$$
\left(\partial_{t} \eta_{\varepsilon}-\kappa \Delta \eta_{\varepsilon}+\rho \sigma_{\varepsilon}, v\right)=\left(g_{\varepsilon}, v\right) \quad \forall v \in H, \text { a.e. in }(0, T) .
$$

We take $v=\sigma_{\varepsilon}$ in the above equation and integrate between $t$ and $t+h$ (with $h \in(0, T-t)$ ) obtaining

$$
\int_{t}^{t+h}\left(\partial_{t} \eta_{\varepsilon}, \sigma_{\varepsilon}\right)+\kappa \int_{t}^{t+h}\left(\nabla \eta_{\varepsilon}, \nabla \sigma_{\varepsilon}\right)+\rho \int_{t}^{t+h}\left\|\sigma_{\varepsilon}\right\|_{H}^{2}=\int_{t}^{t+h}\left(g_{\varepsilon}, \sigma_{\varepsilon}\right) .
$$

Using the fact that $\operatorname{Sign}_{\varepsilon}$ is the Fréchet-differential of $\|\cdot\|_{H, \varepsilon}$, we deduce that

$$
\left(\partial_{t} \eta_{\varepsilon}, \sigma_{\varepsilon}\right)=\frac{\mathrm{d}}{\mathrm{d} t}\left\|\eta_{\varepsilon}\right\|_{H, \varepsilon}
$$

In view of $\underline{3.6}$, we have that

$$
\int_{t}^{t+h}\left(\nabla \eta_{\varepsilon}, \nabla \sigma_{\varepsilon}\right)=\int_{t}^{t+h} \frac{\left\|\nabla \eta_{\varepsilon}\right\|_{H}^{2}}{\max \left\{\varepsilon,\left\|\eta_{\varepsilon}\right\|_{H}\right\}} \geq 0
$$

Finally, as $\left\|\sigma_{\varepsilon}\right\|_{H} \leq 1$ a.e. in $(0, T)$, it follows that

$$
\int_{t}^{t+h}\left(g_{\varepsilon}, \sigma_{\varepsilon}\right) \leq h C_{5}\left(1+\rho^{1 / 2}\right) .
$$

Putting everything together we obtain

$$
\left\|\eta_{\varepsilon}(t+h)\right\|_{H, \varepsilon}-\left\|\eta_{\varepsilon}(t)\right\|_{H, \varepsilon}+\rho \int_{t}^{t+h}\left\|\sigma_{\varepsilon}\right\|_{H}^{2} \leq h C_{5}\left(1+\rho^{1 / 2}\right) .
$$

We remark that $\eta_{\varepsilon}$ converges to $\eta$ in $C^{0}([0, T] ; H)$ as $\varepsilon \rightarrow 0$, (cf. (3.25) $-(3.26)$ ), which can be replicated for the limit as $\varepsilon \rightarrow 0)$. Moreover, the Yosida approximations $\|\cdot\|_{H, \varepsilon}$ converge to $\|\cdot\|_{H}$ uniformly in $H$, by (3.8). It follows that the first two terms of the previous inequality converge. At this point, we take the inferior limit in (6.6) as $\varepsilon \rightarrow 0$ and we use the Lebesgue dominate convergence theorem, property (3.36), and the weak lower semicontinuity of norms obtaining

$$
\|\chi(t+h)\|_{H}-\|\chi(t)\|_{H}+\rho \int_{t}^{t+h}\|\sigma\|_{H}^{2} \leq h C_{5}\left(1+\rho^{1 / 2}\right) .
$$

We divide by $h$ and we take the limit as $h \rightarrow 0$

$$
\frac{\mathrm{d}}{\mathrm{d} t}\left(\|\eta(t)\|_{H}\right)+\rho\|\sigma(t)\|_{H}^{2} \leq C_{5}\left(1+\rho^{1 / 2}\right) \quad \text { for a.a. } t \in(0, T) .
$$

We introduce the function $\psi(t)=\|\eta(t)\|_{H}$ and the quantity

$$
M(\rho)=\rho-C_{5}-C_{5} \rho^{1 / 2} .
$$

We also set $($ see (2.31) $) \psi_{0}=\left\|\vartheta_{0}+\alpha-\eta^{*}\right\|_{H}$. The inequality above implies

$$
\psi^{\prime}(t) \leq-M(\rho), \quad \text { for a.a. } t \text { in }\{t: \psi(t)>0\} .
$$


Using the Young inequality we obtain

$$
M(\rho) \geq \frac{\rho}{2}-C_{5}-\frac{C_{5}^{2}}{2} .
$$

Thus, if we choose

$$
\rho^{*}=2\left(\frac{\psi_{0}}{T}+C_{5}+\frac{C_{5}^{2}}{2}\right),
$$

then for every $\rho>\rho^{*}$ it turns out that $M(\rho)>\psi_{0} / T$.

Finally we can use Lemma 6.1 that guarantees the existence of $T^{*}<T$ such that $\psi$ vanishes in $\left[T^{*}, T\right]$, i.e. the thesis. Moreover, the second part of the Lemma and equation (6.8) lead to

$$
T^{*} \leq \frac{2 \psi_{0}}{\rho-2 C_{5}-C_{5}^{2}}<T .
$$

\subsection{Existence of sliding modes for Problem (B)}

We define for $\varepsilon>0, \chi_{\varepsilon}, g_{\varepsilon}:[0, T] \rightarrow H$ as

$$
\begin{aligned}
\chi_{\varepsilon} & :=\varphi_{\varepsilon}-\varphi^{*}, \\
g_{\varepsilon} & :=\gamma \partial_{t} w_{\varepsilon}-\beta_{\varepsilon}\left(\varphi^{*}\right)-\pi\left(\varphi_{\varepsilon}\right)+\triangle \varphi^{*} .
\end{aligned}
$$

Analogously, we define $\chi=\varphi-\varphi^{*}$. Like in the previous proof, we have that $g_{\varepsilon} \in L^{\infty}(0, T ; H)$ and

$$
\left\|g_{\varepsilon}\right\|_{L^{\infty}(0, T ; H)} \leq C_{10},
$$

where the constant is specified in (2.41). Recalling the definition of $\sigma_{\varepsilon}$, we rewrite equation (3.50) as

$$
\left(\partial_{t} \chi_{\varepsilon}-\triangle \chi_{\varepsilon}+\beta_{\varepsilon}\left(\varphi_{\varepsilon}\right)-\beta_{\varepsilon}\left(\varphi^{*}\right)+\rho \sigma_{\varepsilon}, v\right)=\left(g_{\varepsilon}, v\right) \quad \forall v \in H, \text { a.e. in }(0, T) .
$$

We take $v=\sigma_{\varepsilon}$ in the equation above and integrate between $t$ and $t+h$ (with $h \in(0, T-t)$ ) obtaining

$$
\begin{aligned}
& \int_{t}^{t+h}\left(\partial_{t} \chi_{\varepsilon}, \sigma_{\varepsilon}\right)+\kappa \int_{t}^{t+h}\left(\nabla \chi_{\varepsilon}, \nabla \sigma_{\varepsilon}\right)+\int_{t}^{t+h}\left(\beta_{\varepsilon}\left(\varphi_{\varepsilon}\right)-\beta_{\varepsilon}\left(\varphi^{*}\right), \sigma_{\varepsilon}\right) \\
& +\rho \int_{t}^{t+h}\left\|\sigma_{\varepsilon}\right\|_{H}^{2}=\int_{t}^{t+h}\left(g_{\varepsilon}, \sigma_{\varepsilon}\right) .
\end{aligned}
$$

Inequalities (6.4) and (6.5) are still valid if we substitute $\eta_{\varepsilon}$ with $\chi_{\varepsilon}$. Moreover, using the monotonicity of $\beta_{\varepsilon}$, we have that

$$
\int_{t}^{t+h}\left(\beta_{\varepsilon}\left(\varphi_{\varepsilon}\right)-\beta_{\varepsilon}\left(\varphi^{*}\right), \sigma_{\varepsilon}\right)=\int_{t}^{t+h} \frac{\left(\beta_{\varepsilon}\left(\varphi_{\varepsilon}\right)-\beta_{\varepsilon}\left(\varphi^{*}\right), \chi_{\varepsilon}\right)}{\max \left\{\varepsilon,\left\|\chi_{\varepsilon}\right\|_{H}\right\}} \geq 0 .
$$

Finally, as $\left\|\sigma_{\varepsilon}\right\|_{H} \leq 1$ a.e. in $(0, T)$, we have that

$$
\int_{t}^{t+h}\left(g_{\varepsilon}, \sigma_{\varepsilon}\right) \leq h C_{10} .
$$

Putting everything together we obtain

$$
\left\|\chi_{\varepsilon}(t+h)\right\|_{H, \varepsilon}-\left\|\chi_{\varepsilon}(t)\right\|_{H, \varepsilon}+\rho \int_{t}^{t+h}\left\|\sigma_{\varepsilon}\right\|_{H}^{2} \leq h C_{10} .
$$

For the same considerations made in the previous proof, we may take the limit as $\varepsilon \rightarrow 0$, obtaining

$$
\|\chi(t+h)\|_{H}-\|\chi(t)\|_{H}+\rho \int_{t}^{t+h}\|\sigma\|_{H}^{2} \leq h C_{10} .
$$

We divide by $h$ and, using the Lebesgue differentiation theorem, we take the limit as $h \rightarrow 0$

$$
\frac{\mathrm{d}}{\mathrm{d} t}\|\chi(t)\|_{H}+\rho\|\sigma(t)\|_{H}^{2} \leq C_{10} \quad \text { for a.a. } t \in(0, T) .
$$


We introduce the function $\psi(t)=\|\chi(t)\|_{H}$ and the quantity

$$
M(\rho)=\rho-C_{10} .
$$

We also set $\psi_{0}=\left\|\varphi_{0}-\varphi^{*}\right\|_{H}$ (see (2.42) $)$. Clearly, it holds that

$$
\psi^{\prime}(t) \leq-M(\rho), \quad \text { for a.a. } t \text { in }\{t: \psi(t)>0\},
$$

so we can choose

$$
\rho^{*}=\frac{\psi_{0}}{T}+C_{10},
$$

and consequently $M(\rho)>\psi_{0} / T$, for every $\rho>\rho^{*}$.

Finally we can use Lemma 6.1 that guarantees the existence of $T^{*}<T$ such that $\psi$ vanishes in $\left[T^{*}, T\right]$, i.e. the thesis. Moreover, the second part of the Lemma leads to

$$
T^{*} \leq \frac{\psi_{0}}{\rho-C_{10}}<T .
$$

\section{Acknowledgments}

The current contribution originated from the work done by Davide Manini for the preparation of his master thesis, which has been discussed at the University of Pavia on July 2019. Actually, the paper turns out to offer some extension to the results there contained. The research of Pierluigi Colli is supported by the Italian Ministry of Education, University and Research (MIUR): Dipartimenti di Eccellenza Program (2018-2022) - Dept. of Mathematics "F. Casorati", University of Pavia. In addition, PC gratefully acknowledges some other support from the GNAMPA (Gruppo Nazionale per l'Analisi Matematica, la Probabilità e le loro Applicazioni) of INdAM (Istituto Nazionale di Alta Matematica) and the IMATI C.N.R. Pavia, Italy.

\section{References}

[1] V. Barbu, Nonlinear differential equations of monotone types in Banach spaces, Springer Monographs in Mathematics, Springer, New York, 2010.

[2] V. Barbu, P. Colli, G. Gilardi, G. Marinoschi, and E. Rocca, Sliding mode control for a nonlinear phase-field system, SIAM J. Control Optim., 55 (2017), pp. 2108-2133.

[3] G. Bartolini, L. Fridman, A. Pisano, and E. Usai, eds., Modern sliding mode control theory new perspectives and applications, Lecture Notes in Control and Inform. Sci. 375, Springer, Berlin, 2008.

[4] H. BRÉZIS, Opérateurs maximaux monotones et semi-groupes de contractions dans les espaces de Hilbert, North-Holland, Amsterdam, 1973.

[5] H. BrÉZIS, Functional analysis, Sobolev spaces and partial differential equations, Universitext, Springer, New York, 2011.

[6] G. Caginalp, An analysis of a phase field model of a free boundary, Arch. Rational Mech. Anal., 92 (1986), pp. 205-245.

[7] G. Canevari And P. Colli, Solvability and asymptotic analysis of a generalization of the Caginalp phase field system, Commun. Pure Appl. Anal., 11 (2012), pp. 1959-1982.

[8] G. Canevari and P. Colli, Convergence properties for a generalization of the Caginalp phase field system, Asymptot. Anal., 82 (2013), pp. 139-162.

[9] C. Cavaterra, D. EnĂChescu, and G. Marinoschi, Sliding mode control of the Hodgkin-Huxley mathematical model, Evol. Equ. Control Theory, 8 (2019), pp. 883-902.

[10] M.-B. Cheng, V. Radisavluevic, And W.-C. Su, Sliding mode boundary control of a parabolic PDE system with parameter variations and boundary uncertainties, Automatica J. IFAC, 47 (2011), pp. 381-387. 
[11] P. Colli, G. Gilardi, G. Marinoschi, And E. Rocca, Sliding mode control for a phase field system related to tumor growth, Appl. Math. Optim., 79 (2019), pp. 647-670.

[12] M. Colturato, On a class of conserved phase field systems with a maximal monotone perturbation, Appl. Math. Optim., 78 (2018), pp. 545-585.

[13] G. Dal Maso, An introduction to $\Gamma$-convergence, Progress in Nonlinear Differential Equations and their Applications 8, Birkhäuser Boston, Inc., Boston, MA, 1993.

[14] C. Edwards, E. Fossas Colet, And L. Fridman, eds., Advances in variable structure and sliding mode control, Lecture Notes in Control and Inform. Sci. 334, Springer, Berlin, 2006.

[15] L. C. Evans, Partial differential equations, Grad. Stud. Math. 19, American Mathematical Society, Providence, 1998.

[16] L. Fridman, J. Moreno, And R. Iriarte, eds., Sliding modes after the first decade of the 21st century: state of the art, Lecture Notes in Control and Inform. Sci. 412, Springer, Berlin, 2011.

[17] A. E. Green And P. M. Naghdi, A re-examination of the basic postulates of thermomechanics, Proc. Roy. Soc. London Ser. A, 432 (1991), pp. 171-194.

[18] A. E. GReEn And P. M. NAGhdi, On undamped heat waves in an elastic solid, J. Thermal Stresses, 15 (1992), pp. 253-264.

[19] A. E. Green And P. M. NAGhdi, Thermoelasticity without energy dissipation, J. Elasticity, 31 (1993), pp. 189-208.

[20] L. LevagGI, Infinite dimensional systems' sliding motions, Eur. J. Control, 8 (2002), pp. 508-516.

[21] L. Levaggi, Existence of sliding motions for nonlinear evolution equations in Banach spaces, Discrete Contin. Dyn. Syst., 8 (2013), pp. 477-487.

[22] Y. Orlov And V. I. UtKin, Unit sliding mode control in infinite-dimensional systems, Appl. Math. Comput. Sci., 8 (1998), pp. 7-20. Adaptive learning and control using sliding modes.

[23] Y. V. OrLOv, Discontinuous unit feedback control of uncertain infinite-dimensional systems, IEEE Trans. Automat. Control, 45 (2000), pp. 834-843.

[24] Y. V. ORlov AND V. I. UtKIn, Use of sliding modes in distributed system control problems, Automat. Remote Control, 43 (1983), pp. 1127-1135.

[25] Y. V. Orlov And V. I. UtKin, Sliding mode control in indefinite-dimensional systems, Automatica J. IFAC, 23 (1987), pp. 753-757.

[26] P. Podio-Guidugli, A virtual power format for thermomechanics, Contin. Mech. Thermodyn., 20 (2009), pp. 479-487.

[27] R. T. Rockafellar, Convex analysis, Princeton Mathematical Series, Princeton University Press, Princeton, 1970.

[28] J. Simon, Compact sets in the space $L^{p}(0, T ; B)$, Ann. Mat. Pura Appl. (4), 146 (1987), pp. 65-96.

[29] V. I. Utkin, Sliding modes in control and optimization, Comm. Control Engrg. Ser., Springer, Berlin, 1992.

[30] H. Xing, D. Li, C. GAO, AND Y. KAO, Delay-independent sliding mode control for a class of quasi-linear parabolic distributed parameter systems with time-varying delay, J. Franklin Inst., 350 (2013), pp. 397-418.

[31] K. D. Young And U. ÖZgüner, EDs., Variable structure systems, sliding mode and nonlinear control, Lecture Notes in Control and Inform. Sci. 247, Springer, London, 1999. 